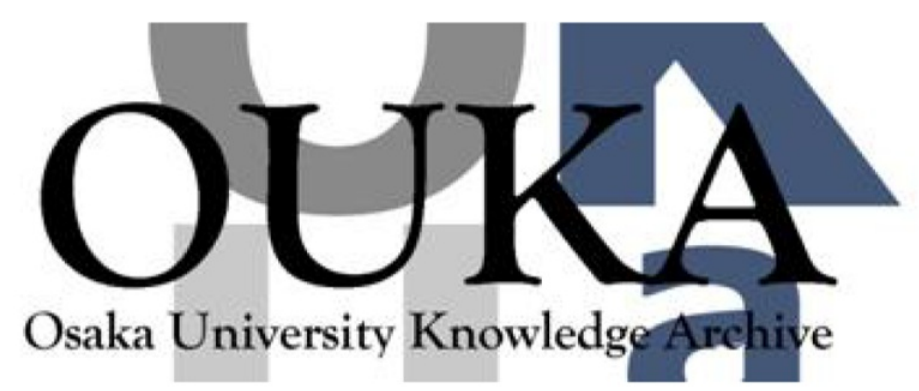

\begin{tabular}{|c|l|}
\hline Title & $\begin{array}{l}\text { Universal extra dimensions after Higgs } \\
\text { discovery }\end{array}$ \\
\hline Author(s) & $\begin{array}{l}\text { Kakuda, Takuya; Nishiwaki, Kenji; Oda, Kinya et } \\
\text { al. }\end{array}$ \\
\hline Citation & Physical Review D. 88(3) p. 035007 \\
\hline Issue Date & $2013-08-06$ \\
\hline oaire:version & VoR \\
\hline URL & https://hdl. handle. net/11094/78750 \\
\hline rights & ○ 2013 American Physical Society \\
\hline Note & \\
\hline
\end{tabular}

Osaka University Knowledge Archive : OUKA

https://ir. Library. osaka-u. ac. jp/

Osaka University 


\title{
Universal extra dimensions after Higgs discovery
}

\author{
Takuya Kakuda, ${ }^{1, *}$ Kenji Nishiwaki, ${ }^{2, \dagger}$ Kin-ya Oda, ${ }^{3, \$}$ and Ryoutaro Watanabe ${ }^{4, \S}$ \\ ${ }^{1}$ Department of Physics, Niigata University, Niigata 950-2181, Japan \\ ${ }^{2}$ Regional Centre for Accelerator-based Particle Physics, Harish-Chandra Research Institute, Allahabad 211 019, India \\ ${ }^{3}$ Department of Physics, Osaka University, Osaka 560-0043, Japan \\ ${ }^{4}$ Theory Group, KEK, Tsukuba, Ibaraki 305-0801, Japan \\ (Received 23 May 2013; published 6 August 2013)
}

\begin{abstract}
We show bounds on five- and six-dimensional universal extra dimension (UED) models from the latest results of the Higgs searches at the LHC and from the electroweak precision data for the $S$ and $T$ parameters. We consider the minimal UED model in five dimensions and the ones in six dimensions, compactified on $T^{2} / Z_{2}, T^{2} /\left(Z_{2} \times Z_{2}^{\prime}\right), T^{2} / Z_{4}, S^{2}, S^{2} / Z_{2}$, the real projective plane, and the projective sphere. The highest possible ultraviolet cutoff scale for each UED model is evaluated from the electroweak vacuum stability by solving the renormalization group equation of the Higgs self-coupling. This scale turns out to be lower than the conventional one obtained from the perturbativity of the gauge coupling. The resultant $95 \%$ C.L. lower bounds on the first Kaluza-Klein scale from the LHC results and from the $S, T$ analysis are 600 and $700 \mathrm{GeV}$ in the minimal UED model, while those in the sixdimensional UED models are $800-1300 \mathrm{GeV}$ and $900-1500 \mathrm{GeV}$, respectively.
\end{abstract}

DOI: 10.1103/PhysRevD.88.035007

PACS numbers: $12.60 .-\mathrm{i}, 11.10 . \mathrm{Kk}$

\section{INTRODUCTION}

The ATLAS and CMS experiments at the CERN LHC have observed a particle around $126 \mathrm{GeV}$, which is consistent with the Standard Model (SM) Higgs boson [1,2]. The signal strength (defined as the ratio of the production cross section times the branching ratio of the observed particle to that of the SM Higgs) for the decay channels into diphoton $(\gamma \gamma)$ and diboson $(Z Z$ and $W W)$ are reported in Refs. [3-8]. Namely, the signal strengths of $H \rightarrow \gamma \gamma$, $Z Z$, and $W W$ have turned out to be $1.65 \pm 0.24_{-0.18}^{+0.25}$, $1.7_{-0.4}^{+0.5}$, and $1.01 \pm 0.31$ at the ATLAS experiment, and $0.78 \pm 0.27$ (multivariate analysis based), $0.91_{-0.24}^{+0.30}$, and $0.71 \pm 0.37$ (cut based) at the CMS experiment, respectively. All these results are consistent with the SM within less than $2 \sigma$, but there still remains room for a new physics effect. See, e.g., Refs. [9-16] for analyses based on effective Lagrangian methods. ${ }^{1}$ In this paper, we study the constraints on the existence of the universal extra dimensions (UEDs) $[18]^{2}$ from the Higgs searches.

In the UED scenario, each SM field propagates in one or more compactified extra dimensions and is accompanied by its massive copies, called Kaluza-Klein (KK) particles. Already in the simplest five-dimensional (5D) minimal UED (mUED) model on the orbifold $S^{1} / Z_{2}$ [18], in which no tree-level brane-localized term is assumed at an UV

\footnotetext{
*kakuda@muse.sc.niigata-u.ac.jp

†nishiwaki@hri.res.in

‡odakin@phys.sci.osaka-u.ac.jp

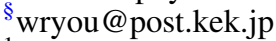

${ }^{1}$ Global fits of the minimal UED scenario were done in Ref. [17].

${ }^{2}$ See also Ref. [19] for an earlier proposal of a TeV scale extra dimension.
}

cutoff scale of the 5D gauge theory, there exists an attractive feature: The lightest KK particles become stable due to the symmetry in the geometry, the KK parity, and serves as a natural dark matter candidate [20,21]. The KK particles are expected to exist above around $1 \mathrm{TeV}$, which is consistent to the indirect bound from the $S, T$ parameters, $M_{\mathrm{KK}} \gtrsim 700 \mathrm{GeV}$ [22], and from the $b \rightarrow s \gamma$ process, $M_{\mathrm{KK}} \gtrsim 600 \mathrm{GeV}$ [23], with $M_{\mathrm{KK}}$ being the first KK mass. The prospects of the mUED at the LHC and future linear colliders have been discussed in Refs. [24-43] and those in the context of discrimination from other models with similar final states in Refs. [44-49].

A way of extending the minimal scenario is to consider the model in two-dimensional extra space. Models have been proposed on two-torus, $T^{2} / Z_{2}$ [18], $T^{2} / Z_{4}$ (chiral square) [50,51], $T^{2} /\left(Z_{2} \times Z_{2}^{\prime}\right)$ [52], on two-sphere $S^{2} / Z_{2}$ [53], on $S^{2}$ with a Stueckhelbarg field [54,55], and on the nonorientable manifolds: the real projective plane $R P^{2}$ [56] and the projective sphere (PS) [57]. An advantage of such a six-dimensional (6D) UED model is that the number of generation is predicted to be (a multiple of) three [58], from the requirement of the cancellation of the $6 \mathrm{D}$ gravitational and $S U(2)_{L}$ global anomalies, which cannot be eliminated via the Green-Schwarz mechanism. We can find works on collider phenomenology in the cases of $T^{2} / Z_{4}$ [59-64] and of $R P^{2}$ [65-68]. Recently, other possibilities of generalization of these models by an introduction of the bulk mass term and/or the brane-localized Lagrangians have been studied in Refs. [69-81].

In general, the single Higgs production process via the loop-induced gluon fusion is enhanced in a UED model, while the branching ratio of the Higgs to diphoton is suppressed because of interference effect between bosons and fermions inside the loops. These UED effects have been 
first shown in the 5D mUED [82]. In 6D UED, the enhancement of the gluon fusion is studied in Ref. [83], and the diphoton decay rate is obtained in Ref. [84]. See Refs. [54,55,85-87] for more works in this direction. In this paper, we perform more elaborated analysis compared with our previous work in Ref. [84], with a varying portion of production channels for each event category. We also estimate constraints from the $S$ and $T$ parameters in every model for completeness. In addition to the effects of the KK Higgs boson and the KK top quark [22,88], those of the KK gauge boson are taken into account for the first time in the literature.

An important number in the UED phenomenology is the highest possible UV cutoff scale, $\Lambda_{\max }$, allowed by the electroweak vacuum stability. The cutoff scale $\Lambda$ of a UED model gives the upper bound of the KK summation in loop processes. Therefore, different values of $\Lambda$ result in different bounds on $M_{\mathrm{KK}}$. In the mUED with the $126 \mathrm{GeV}$ Higgs, the highest possible $\Lambda$ becomes quite low, $\Lambda \lesssim 5 M_{\mathrm{KK}}$ [89]. In this paper, we examine all the 5D and 6D UED models without resorting to the approximation employed in the analysis of gauge coupling running in our previous work [54]. When we consider a model with a low cutoff scale, threshold corrections via higher-dimensional operators can become significant, which we will take into account for the $S$ and $T$ parameter constraints. Effects from such higherdimensional operators on Higgs signals have already been discussed in Ref. [84].

The paper is organized as follows. In Sec. II, we estimate the highest cutoff scale of all the UED models. Based on the results, we calculate the direct and indirect bounds from the LHC results in Sec. III and from the $S, T$ parameters in Sec. IV. In Sec. V, we summarize our results and discuss future prospects. Detailed formulas that we use in this paper can be found in the appendix.

\section{VACUUM STABILITY BOUND}

In this section, we estimate the UV cutoff scale in seven types of six-dimensional UED models on $T^{2} / Z_{2}, T^{2} /\left(Z_{2} \times\right.$ $\left.Z_{2}^{\prime}\right), T^{2} / Z_{4}, R P^{2} ; S^{2}$ with a Stueckhelbarg field, $S^{2} / Z_{2}$; and the PS and in the 5D mUED. The orbifolding in $T^{2} / Z_{2}$, $T^{2} /\left(Z_{2} \times Z_{2}^{\prime}\right)$ and $T^{2} / Z_{4}$ on two-torus $\left(T^{2}\right)$ projects out a chiral zero mode from each matter fermion. On two-sphere $S^{2}$, we can obtain a Weyl fermion in each zero mode, due to the monopolelike classical configuration of an extra $U(1)_{X}$ gauge boson. However, we must eliminate the phenomenologically unacceptable massless zero mode of the $U(1)_{X}$ gauge boson, and we will discuss three possibilities for treating this issue in this paper.

The geometries of $R P^{2}$ and PS are unoriented and have no local fixed points. Consequently, their KK mass spectra take unique forms, for which the pattern is distinctive from those of the other UED models. A brief review of the models studied in this paper can also be found in our previous paper [55].
To find the highest possible UV cutoff $\Lambda_{\max }$, we examine the vacuum stability bound by solving the renormalization group equation (RGE). As said above, the UV cutoff plays an important role in the estimation of the KK loop effects in processes involving loop diagrams. In later sections, we use the results of this section for calculating the deviations in the single Higgs production processes and the Peskin-Takeuchi $S$ and $T$ parameters. It is noted that, in this paper, we mainly consider the situation where radii of compactified fifth and sixth directions $R_{5}, R_{6}$ are the same: $R_{5}=R_{6}=R .^{3}$

\section{A. RGE in 6D UED models}

Considering the RGE is an effective way of probing scale dependence. Its concrete form is derived from the invariance, under the change of the renormalization scale $\mu$, of the bare vertex function $\Gamma_{0}$, which is a function of bare parameters. The scale invariance requires that

$$
\mu \frac{d}{d \mu} \Gamma_{0}\left(\left\{c_{0},\left\{m_{0}\right\},\left\{\Phi_{0}\right\}\right)=0,\right.
$$

where $\left\{c_{0}\right\},\left\{m_{0}\right\}$, and $\left\{\Phi_{0}\right\}$ represent sets of bare couplings, masses, and fields, respectively. Since bare parameters and fields are divergent themselves, we can rewrite the bare ones with finite physical ones (renormalized parameters and fields) and counter terms, which contain divergences. In this paper, we show all the bare/renormalized variables with/without the subscript " 0. ."

In the following, we consider the RGE for the Higgs quartic coupling $\lambda$ in the 6D models. We obey the convention of Ref. [91] in describing the electroweak (EW) sector. The potential of the Higgs field $H$ at the tree level is depicted as

$$
-\frac{M_{H 0}^{2}}{2} H_{0}^{2}+\frac{\lambda_{(6) 0}}{4} H_{0}^{4}
$$

where $M_{H 0}$ and $\lambda_{(6) 0}$ are the bare Higgs mass and 6D Higgs couplings. After the $6 \mathrm{D}$ bare Higgs field $H_{0}$ is KK expanded, we can find the zero mode $H_{0}^{(0)}$, where we use a superscript for a KK index. In considering the one-loop running of $\lambda$, we need not consider the renormalization of the Higgs mass, and hence the physical Higgs mass $m_{H}$ becomes

$$
m_{H}=\sqrt{\lambda_{(6) 0}} v_{(6) 0}=\sqrt{\lambda_{(6)}} \boldsymbol{v}_{(6)}=\sqrt{\lambda} \boldsymbol{v},
$$

where the four-dimensional (4D) Higgs vacuum expectation value $v=246 \mathrm{GeV}$ and quartic coupling $\lambda$ are expressed as $v_{(6)}=v / \sqrt{V}, \lambda_{(6)}=\lambda \sqrt{V}$, with $V$ being the volume of the extra dimensions. Let us write

\footnotetext{
${ }^{3}$ In the $T^{2} / Z_{4}$ model, the condition $R_{5}=R_{6}=R$ is automatically realized due to the property of the orbifolding. See also Ref. [90] for a realization of $C P$ violation from the complex structure of $T^{2} / Z_{4}$, which appears in four-dimensional effective interactions after $\mathrm{KK}$ decomposition.
} 


$$
H_{0}^{(0)}=\sqrt{Z_{H}} H^{(0)}, \quad \lambda_{0}=Z_{\lambda}\left(Z_{H}\right)^{-2} \lambda,
$$

where $Z_{\lambda}$ is the renormalization factor for the Higgs quartic coupling and $\sqrt{Z_{H}}$ is that for the wave function renormalization of the Higgs zero mode. We also need the information of the RGEs for the gauge and Yukawa couplings to compute the running of $\lambda$. We summarize the beta functions,

$$
\mu \frac{d}{d \mu} \mathcal{Q}=\beta_{\mathcal{Q}}
$$

where detailed form of $\beta_{Q}$ can be found in Appendix A.

Let us review how to compute RGEs in a theory with (a) compactified extra dimension(s). We adopt the bottom-up approach discussed in Refs. [92,93], which takes into account a contribution of a massive particle to the beta functions when the increasing scale $\mu$ passes its mass. In the case of the UED, after KK decomposition, the corresponding 4D effective theory contains not only the SM fields but also their KK partners. Following this prescription, we get

$$
\beta_{\mathcal{Q}}=\beta_{Q}^{(\mathrm{SM})}+\sum_{s: \text { massuve states }} \theta\left(\mu-M_{s}\right)\left(N_{s} \beta_{s, \mathcal{Q}}^{(\mathrm{NP})}\right)
$$

where $\beta_{Q}^{(\mathrm{SM})}$ and $\beta_{s, \mathcal{Q}}^{(\mathrm{NP})}$ are the contributions from the SM particles and from the new massive ones with mass $M_{s}$, respectively, and $N_{s}$ is the number of degenerated states. At the tree level, $M_{s}$ is expressed as

$$
M_{s}^{2}=m_{s,(\mathrm{SM})}^{2}+M_{s,(\mathrm{KK})}^{2},
$$

where $m_{s,(\mathrm{SM})}$ is the SM mass of the corresponding zero mode, and $M_{s,(\mathrm{KK})}$ are KK masses. In general, the value of $M_{s,(\mathrm{KK})}$ is much greater than that of $m_{s,(\mathrm{SM})}$, and $M_{s}^{2}$ can be approximated as $M_{s}^{2} \simeq M_{s,(\mathrm{KK})}^{2}$.

Let us review the KK expansions in the 6D UED models. In the models on the orbifolded $T^{2}$ [namely, $T^{2} / Z_{2}$, $T^{2} /\left(Z_{2} \times Z_{2}^{\prime}\right)$, and $\left.T^{2} / Z_{4}\right]$ and the one on $R P^{2}$, the $\mathrm{KK}$ mass $M_{s,(\mathrm{KK})}^{2}$ becomes of $T^{2}$ type:

$$
M_{s,(\mathrm{KK})}^{2} \rightarrow M_{(m, n)}^{2}:=\frac{m^{2}}{R_{5}^{2}}+\frac{n^{2}}{R_{6}^{2}},
$$

where $m(n)$ is the KK index along the fifth (sixth) direction, and $N_{(m, n)}=1$ irrespective of $m$ and $n$. We note that the beta functions for gauge, Yukawa, and Higgs selfcouplings take the same forms irrespective of the models based on $T^{2}$ and are independent of the KK indices. This reason is as follows. Because of the flat profile of the zero modes, the three-point functions with one SM field and the four-point functions with two SM fields become universal at their leading order after using the orthonormality of KK mode functions. In contrast, the value $N_{s}$ and the summation of the KK index $\sum_{s}$ in Eq. (6) are affected by the difference in the patterns of the orbifolding. Hence, the evolution of $\lambda$ depends on the choice of the model. The explicit range of $m, n$ summation is shown in Table I.
TABLE I. The range of the parameters $(m, n)$ except for the zero mode $(m, n)=(0,0)$ in each case of the orbifolding.

\begin{tabular}{lc}
\hline \hline Type of orbifolding & Range of $(m, n)$ \\
\hline$T^{2} / Z_{2}$ & $m+n \geq 1$, or $m=-n \geq 1$ \\
$T^{2} /\left(Z_{2} \times Z_{2}^{\prime}\right)$ & $0 \leq m<\infty, 0 \leq n<\infty ;(m, n) \neq(0,0)$ \\
$T^{2} / Z_{4}$ & $1 \leq m<\infty, 0 \leq n<\infty$ \\
\hline \hline
\end{tabular}

Let us turn to the model on $R P^{2}$. In Fig. 1, we show the surviving modes. The surviving modes of KK fermions become the same as in the $T^{2} / Z_{2}$ model. On the other hand, the patterns of the bosonic particles are complicated. The allowed range of $m$ and $n$ is $m \geq 0, n \geq 0$, and the type of surviving mode is classified into the following four. In region $\mathrm{I}, \quad(m, n)=(0,2),(0,4),(0,6), \ldots$ and $(m, n)=$ $(2,0),(4,0),(6,0), \ldots$; a physical scalar mode coming from the extra component of the $6 \mathrm{D}$ gauge boson is projected out. In region II, $(m, n)=(0,1),(0,3)$, $(0,5), \ldots$ and $(m, n)=(1,0),(3,0),(5,0), \ldots$; the only surviving bosonic mode is this scalar that was projected in region I. In region III, $m \geq 1, n \geq 1$; all the bosonic modes are left as is, just like in other orbifolded models on $T^{2}$. In the last region IV, only fermionic degrees of freedom remain.

Next, we go on to the models based on $S^{2}$. The explicit form of the KK mass $M_{s,(\mathrm{KK})}^{2}$ on $S^{2}$ is

$$
M_{s,(\mathrm{KK})}^{2} \rightarrow M_{(j)}^{2}:=\frac{j(j+1)}{R^{2}},
$$

with the index $j \geq 1$. For each $j$ th mode in the $S^{2}, S^{2} / Z_{2}$, and PS models, respectively, the number of degrees of freedom reads

$$
\begin{aligned}
& n^{S^{2}}(j)=2 j+1 \\
& n^{S^{2} / Z_{2}}(j)=\left\{\begin{array}{ll}
j+1 & \text { for } j=\text { even } \\
j & \text { for } j=\text { odd }
\end{array},\right. \\
& n_{\text {fermion }}^{\mathrm{PS}}=2 j+1, \quad n_{\mathrm{even}}^{\mathrm{PS}}(j)=\left\{\begin{array}{l}
2 j+1 \\
0
\end{array},\right. \\
& n_{\text {odd }}^{\text {PS }}(j)=\left\{\begin{array}{ll}
0 & \text { for } j=\text { even } \\
2 j+1 & \text { for } j=\text { odd }
\end{array} .\right.
\end{aligned}
$$

In the cases of $S^{2}$ and $S^{2} / Z_{2}$, the number of the surviving degrees of freedom is the same for KK bosons and fermions. On the other hand, the PS is similar to $R P^{2}$; that is, surviving KK bosons are divided into two categories, even and odd. The even category includes all the KK bosons except for the physical scalar from the 6D gauge boson, while the odd one only contains this one. We note that the number of degenerate states is $2 j+1$, irrespective of the statistics of the particles and their oddness/evenness. Finally, we comment on the beta functions of the $S^{2}$-based models. From the surviving bosonic particles in 
KAKUDA et al.
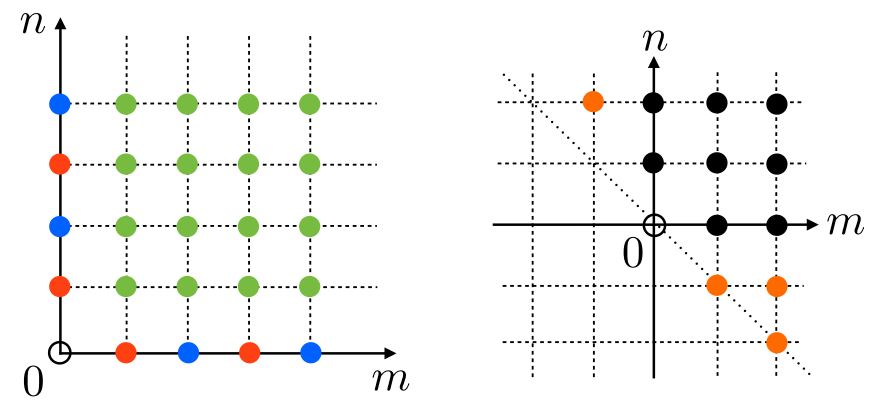

FIG. 1 (color online). Left: The patterns of the remaining bosonic modes in the $R P^{2}$ model, where blue, red, and green points indicate that they belong to the regions I, II, and III, respectively. The definitions of these regions are found in the text. Right: The same as left one for fermonic modes. At the orange points (region IV), there are no bosonic modes. In both pictures, the black circles $(m=n=0)$ correspond to the SM particles.

each KK level, we can see that the RGEs in $S^{2}, S^{2} / Z_{2}$ are similar to those in $T^{2} / Z_{2}, T^{2} /\left(Z_{2} \times Z_{2}^{\prime}\right), T^{2} / Z_{4}$, while those in the PS are similar to those in $R P^{2}$.

\section{B. Running of higgs self-coupling and vacuum stability}

Following the discussion in the previous section, we evaluate the constraints on the highest possible UV cutoff scale $\Lambda$ from the vacuum stability of the Higgs potential. In our analysis, we literally evaluate the KK summation in Eq. (6), unlike the previous analysis in Ref. [55], in which we obtained the UV cutoff scale of the UED models from the perturbativity of the 4D gauge couplings via the RGEs with its KK summation replaced by an integration. In other words, we treat the threshold correction when the reference energy crosses the mass of a KK particle explicitly in our numerical calculation. As it was discussed in the previous section, we can ignore the mass coming from the Higgs mechanism with good precision. Here, we adopt the following criterion for determining $\Lambda_{\max }$ :
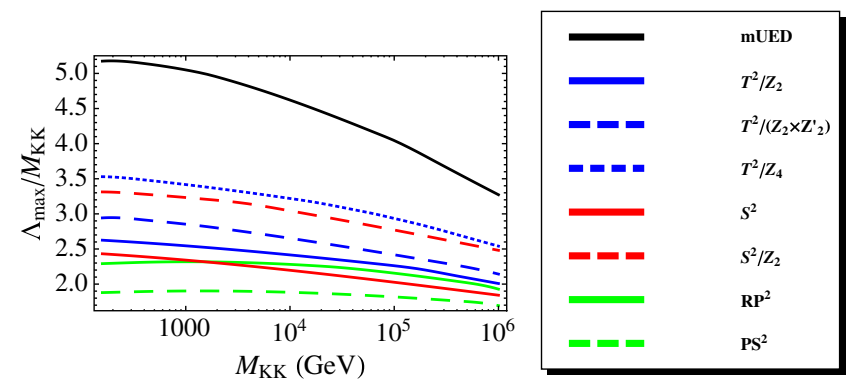

FIG. 2 (color online). Left: Upper bounds on the UV cutoff of the UED models as a function of $M_{\mathrm{KK}}$, with the initial conditions in Eq. (14). Right: Our color convention for types of the UED models. The lines in red, blue, and green show the results of $T^{2}$-based, $S^{2}$-based, and nonorientable-manifold-based UEDs, respectively.
PHYSICAL REVIEW D 88, 035007 (2013)

TABLE II. Upper bounds on cutoff scale $\Lambda_{\max }=\tilde{\Lambda}_{\max } M_{\mathrm{KK}}$ with $M_{\mathrm{KK}}=1 \mathrm{TeV}$ and the initial conditions in Eq. (14).

\begin{tabular}{ccccccccc}
\hline \hline \multicolumn{1}{c}{$T^{2} /$} \\
Model & mUED & $T^{2} / Z_{2}$ & $\left(Z_{2} \times Z_{2}^{\prime}\right)$ & $T^{2} / Z_{4}$ & $S^{2}$ & $S^{2} / Z_{2}$ & $R P^{2}$ & PS \\
\hline$\tilde{\Lambda}_{\max }$ & 5.0 & 2.5 & 2.9 & 3.4 & 2.3 & 3.2 & 2.3 & 1.9 \\
\hline \hline \multicolumn{8}{c}{$\lambda\left(\mu=\Lambda_{\max }\right)=0}$, &
\end{tabular}

where the Higgs potential is destabilized.

We note that the vacuum stability bound is sensitive to the differences in the initial condition of the Higgs selfcoupling $\lambda$ and the top Yukawa coupling $y_{t}$ [94,95]. In our analysis, we adopt the following values:

$$
\begin{aligned}
& \frac{v^{2}}{2} \lambda\left(\mu=m_{Z}\right)=126^{2} \mathrm{GeV}^{2}, \\
& \frac{v}{\sqrt{2}} y_{t}(\mu=173.5 \mathrm{GeV})=160 \mathrm{GeV},
\end{aligned}
$$

where $m_{Z}$ is the $\mathrm{Z}$-boson mass, the $126 \mathrm{GeV}$ is the observed Higgs mass at the LHC, the $173.5 \mathrm{GeV}$ and the $160 \mathrm{GeV}$ are the latest values of the pole and the $\overline{\mathrm{MS}}$ masses of the top quark reported by the particle data group [96], respectively.

The results are summarized in Fig. 2, and the values with $M_{\mathrm{KK}}=1 \mathrm{TeV}$ are also listed in Table II. $M_{\mathrm{KK}}$ means the first KK mass: $M_{\mathrm{KK}}=1 / R$ for the $S^{1} / Z_{2}$ (mUED) and $T^{2}$-based compactifications (namely, $T^{2} / Z_{2}, T^{2} /\left(Z_{2} \times Z_{2}^{\prime}\right.$ ), $T^{2} / Z_{4}$ and $R P^{2}$ ) and $M_{\mathrm{KK}}=\sqrt{2} / R$ for the $S^{2}$-based ones (namely, $S^{2} / Z_{2}$, PS and $S^{2}$ ), where we assume $R_{5}=R_{6}=$ $R$. It is noted that the mUED case has been studied in Refs. [97-100] in many contexts, and we find a study in the case of $T^{2} / Z_{4}[101] .{ }^{4}$ We mention that our conclusion on the mUED is consistent with that in a previous analysis in Ref. [100]. The constraints from vacuum stability, shown in Table II, are tighter than our previous bounds from perturbativity of the gauge couplings: $\Lambda_{\max } \sim 5 M_{\mathrm{KK}}$ in $T^{2} / Z_{2}, T^{2} / Z_{2} \times Z_{2}^{\prime}, T^{2} / Z_{4}, R P^{2}$ and $\Lambda_{\max } \sim 7 M_{\mathrm{KK}}$ in $S^{2}, S^{2} / Z_{2}$, PS. We note that, in the previous analysis, we ignored differences in types of the compactifications and did not put a bound on the mUED since the KK summations in the single Higgs production and the Higgs decay, which are important in LHC phenomenology and which we consider in the next section, are convergent in this case.

Next, we consider the effects when we change the values of top Yukawa coupling in the initial conditions of the RGEs with $M_{\mathrm{KK}}=1 \mathrm{TeV}$. We note that, within the SM, various values of $\overline{\mathrm{MS}}$ top mass $\left.m_{t}\right|_{\overline{\mathrm{MS}}}$ have been reported between 160 and $175 \mathrm{GeV}$ [94-96,104]. Based on this fact, we calculate the bounds on $\lambda$ with varying the initial condition of the top Yukawa as

\footnotetext{
${ }^{4} \mathrm{We}$ can find some related works on the evolutions of a higherdimensional neutrino operator in the mUED [89,102] and in the $T^{2} / Z_{4}$ [101] and of the Cabibbo-Kobayashi-Maskawa matrix [103] in the mUED context.
} 


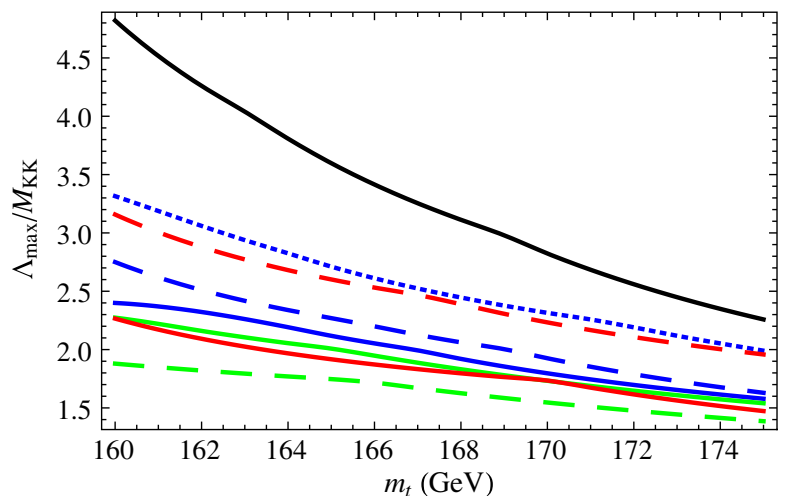

FIG. 3 (color online). Cutoff upper bounds on the 6D UED models and the mUED with the initial condition of the Higgs in Eq. (14) and of the top quark in Eq. (15) with changing in the region of [ $160 \mathrm{GeV}, 175 \mathrm{GeV}$ ] with $M_{\mathrm{KK}}=1 \mathrm{TeV}$. Conventions of line colors and shapes are the same as in Fig. 2.

$$
\begin{aligned}
& \frac{v}{\sqrt{2}} y_{t}(\mu=173.5 \mathrm{GeV})=\left.m_{t}\right|_{\overline{\mathrm{MS}}}, \\
& \quad \text { for } 160 \mathrm{GeV} \leq\left. m_{t}\right|_{\overline{\mathrm{MS}}} \leq 175 \mathrm{GeV} .
\end{aligned}
$$

Our result, depicted in Fig. 3, is sensitive to the value of $\left.m_{t}\right|_{\overline{\mathrm{MS}}}$ and is consistent with the analyses in Ref. [99] (mUED) and in Ref. [101] $\left(T^{2} / Z_{4}\right)$. We cannot avoid the ambiguity originating from the top Yukawa coupling. From Figs. 2 and 3, we find that the dependence of $\Lambda$ on $M_{\mathrm{KK}}$ and $\left.m_{t}\right|_{\overline{\mathrm{MS}}}$ is greater in the mUED than in the 6D UED models. In the latter, the KK threshold corrections are larger than those in the mUED because of their denser KK spectra, and hence the vacuum becomes unstable at a lower energy scale.

\section{HIGGS SIGNALS AT LARGE HADRON COLLIDER}

Equipped with the knowledge for the cutoff scale of UED models in the previous section, we estimate the bound on their KK mass scale from the recent results of Higgs search at the LHC.

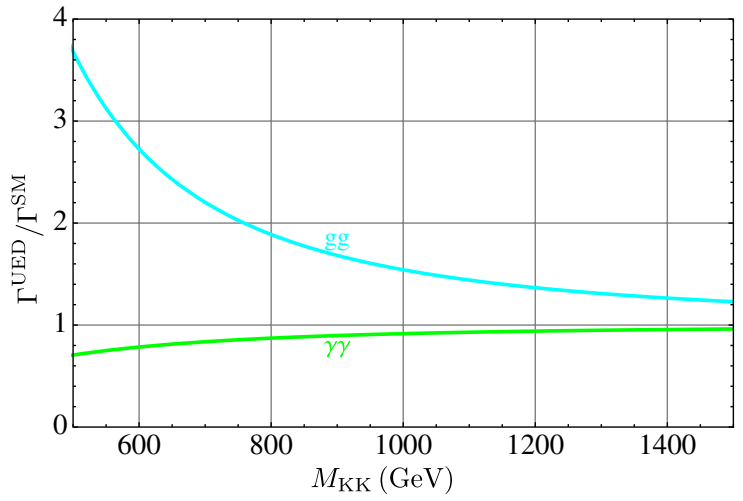

\section{A. Feature of higgs signals in UED models}

The structure of the Higgs signal at the LHC can be divided into the production and decay. The Higgs production is dominated by the gluon fusion process $g g \rightarrow H$, which is induced by the top loop. One of the most important Higgs decay channels that lead to its discovery is the diphoton one $H \rightarrow \gamma \gamma$, which is induced by the top and $W$ boson loops. The Higgs signal is very sensitive to the contribution of the loop corrections at the LHC. In UED models, a lot of additional KK loops contribute to both $g g \rightarrow H(H \rightarrow g g)$ and $H \rightarrow \gamma \gamma$. The KK top loop contribution to the gluon fusion production cross section takes the following form:

$$
\begin{gathered}
\hat{\sigma}_{g g \rightarrow H}^{\mathrm{UED}}=\frac{\pi^{2}}{8 m_{H}} \Gamma_{H \rightarrow g g}^{\mathrm{UED}} \delta\left(\hat{s}-m_{H}^{2}\right), \\
\Gamma_{H \rightarrow g g}^{\mathrm{UED}}=K \frac{\alpha_{s}^{2}}{8 \pi^{2}} \frac{m_{H}^{3}}{v_{\mathrm{EW}}^{2}}\left|J_{t}^{\mathrm{SM}}\left(m_{H}^{2}\right)+J_{t}^{\mathrm{KK}}\left(m_{H}^{2}\right)\right|^{2},
\end{gathered}
$$

where $K \sim 1.5$ is the $\mathrm{K}$ factor accounting for the higherorder QCD corrections for the case of the LHC, $\alpha_{s}=\frac{g_{s}^{2}}{4 \pi}$ is the fine structure constant for $\mathrm{QCD}, v \simeq 246 \mathrm{GeV}$ is the electroweak scale, and $J_{t}^{\mathrm{SM} / \mathrm{KK}}$ denotes the $\mathrm{SM} / \mathrm{KK}$ top quark loop function, defined in Refs. [54,55]. The KK top quark and $\mathrm{KK} W$-boson loop contributions to the Higgs decay into diphoton are written as

$$
\begin{aligned}
\Gamma_{H \rightarrow \gamma \gamma}^{\mathrm{UED}}= & \frac{\alpha^{2} G_{F} m_{H}^{3}}{8 \sqrt{2} \pi^{3}} \mid J_{W}^{\mathrm{SM}}\left(m_{H}^{2}\right)+J_{W}^{\mathrm{KK}}\left(m_{H}^{2}\right) \\
& +\left.\frac{4}{3}\left(J_{t}^{\mathrm{SM}}\left(m_{H}^{2}\right)+J_{t}^{\mathrm{KK}}\left(m_{H}^{2}\right)\right)\right|^{2},
\end{aligned}
$$

where $\alpha=\frac{e^{2}}{4 \pi}$ and $G_{F}$ are fine structure constants for the QED and Fermi constant, respectively. The SM/KK $W$-boson loop functions $J_{W}^{\mathrm{SM} / \mathrm{KK}}$ are defined in Ref. [54]. We have listed them in Appendix B.

Because of these additional contributions, the loopinduced processes $g g \rightarrow H \quad(H \rightarrow g g)$ and $H \rightarrow \gamma \gamma$

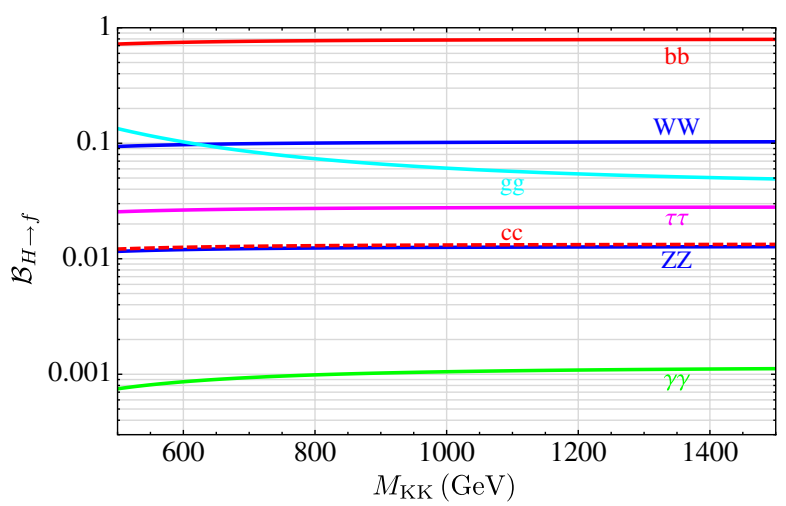

FIG. 4 (color online). For the Higgs decay in the $T^{2} / Z_{2}$ UED model, we show the UED/SM ratio (left) and the branching ratio (right) as a function of $M_{\mathrm{KK}}$ for each final state, which is indicated by the caption within figure; especially we distinguish the almost degenerate $c c$ and $Z Z$ by dashed and solid lines, respectively. 
TABLE III. The ATLAS result of $H \rightarrow \gamma \gamma$ analysis. The ATLAS experiment defines these event categories and uses these ratios of the production channels as in Ref. [3].

\begin{tabular}{lcrrrrr}
\hline \hline$I$ & $\begin{array}{c}\mu_{H \rightarrow \gamma \gamma}^{I} \\
\text { Event category }\end{array}$ & \multicolumn{4}{c}{$\epsilon_{\gamma \gamma}^{I, X}(\%)$} & \\
Signal strength & \multicolumn{1}{c}{$\mathrm{GF}$} & $\mathrm{VBF}$ & $\mathrm{VH}(\mathrm{WH})$ & $\mathrm{VH}(\mathrm{ZH})$ & $\mathrm{ttH}$ \\
\hline Unconventional central low $P_{T}$ & $0.9 \pm 0.7$ & 93.7 & 4.0 & 1.4 & 0.8 & 0.2 \\
Unconventional central high $P_{T}$ & $1.0_{-0.9}^{+1.1}$ & 79.3 & 12.6 & 4.1 & 2.5 & 1.4 \\
Unconventional rest low $P_{T}$ & $2.6_{-1.0}^{+0.9}$ & 93.2 & 4.0 & 1.6 & 1.0 & 0.1 \\
Unconventional rest high $P_{T}$ & $2.7_{-1.2}^{+1.3}$ & 78.1 & 13.3 & 4.7 & 2.8 & 1.1 \\
Conventional central low $P_{T}$ & $1.4_{-0.9}^{+1.0}$ & 93.6 & 4.0 & 1.3 & 0.9 & 0.2 \\
Conventional central high $P_{T}$ & $2.0_{-1.3}^{+1.5}$ & 78.9 & 12.6 & 4.3 & 2.7 & 1.5 \\
Conventional rest low $P_{T}$ & $2.2_{-1.0}^{+1.2}$ & 93.2 & 4.1 & 1.6 & 1.0 & 0.1 \\
Conventional rest high $P_{T}$ & $1.3 \pm 1.3$ & 77.7 & 13.0 & 5.2 & 3.0 & 1.1 \\
Conventional transition & $2.8_{-1.6}^{+1.7}$ & 90.7 & 5.5 & 2.2 & 1.3 & 0.2 \\
Loose high mass 2 jet & $2.8_{-1.4}^{+1.7}$ & 45.0 & 54.1 & 0.5 & 0.3 & 0.1 \\
Tight high mass 2 jet & $1.6_{-0.6}^{+0.8}$ & 23.8 & 76.0 & 0.1 & 0.1 & 0.0 \\
Low mass 2 jet & $0.3_{-1.5}^{+1.7}$ & 48.1 & 3.0 & 29.7 & 17.2 & 1.9 \\
$E_{T}^{\text {miss significance }}$ & $3.0_{-1.9}^{+2.7}$ & 4.1 & 0.5 & 35.7 & 47.6 & 12.1 \\
One lepton & $2.7_{-1.7}^{+2.0}$ & 2.2 & 0.6 & 63.2 & 15.4 & 18.6 \\
\hline \hline
\end{tabular}

receive nontrivial effects, which we compute and use to estimate the branching ratios and the Higgs decay rates into the diphoton and digluon. As an illustration, we show results for the $T^{2} / Z_{2}$ model in Fig. 4. The UED/SM ratio of $H \rightarrow g g$ is always enhanced while that of $H \rightarrow \gamma \gamma$ is suppressed as already seen in Ref. [54]. These behaviors also affect the branching ratios of the Higgs decay as shown in the right panel of Fig. 4. The enhancement in $H \rightarrow g g$ is straightforwardly understood as the $\mathrm{KK}$ top contributions in the loop diagram. The reason for the suppression in $H \rightarrow \gamma \gamma$ is as follows. Since the vectorlike fermions have twice the degrees of freedom compared with SM fermions, their negative contributions to the Higgs decay rate become larger than the positive ones coming from the KK W loop. Thus, the sum of the KK loops becomes negative, and it overcomes the positive SM contribution. As a consequence, the decay rate of $H \rightarrow \gamma \gamma$ is suppressed compared with the SM.

\section{B. Strategy to constrain the KK mass scale}

As shown above, the UED models give different production cross sections in the gluon fusion (GF). On the other hand, the other productions, which are the vector boson fusion (VBF), the Higgs-strahlung (VH), and the associated production with a $t \bar{t}$ pair $(\mathrm{ttH})$, are the same as the SM. We express the $\mathrm{VH}$ production associated with $W$ and $Z$ by WH and $\mathrm{ZH}$, respectively. In the recent analysis, the ATLAS and CMS experiments have reported on ratios of these production channels in $H \rightarrow \gamma \gamma, Z Z$, and $W W$ for each category tagging their decays [3-8]. ${ }^{5}$ Such ratios are quite important

\footnotetext{
${ }^{5}$ We use " $Z Z$ " and " $W W$ " as the meaning of $Z Z \rightarrow 4 \ell$ and $W W \rightarrow 2 \ell 2 \nu$ for simplicity.
}

for obtaining the bound on UED models because of the nontrivial effect of the KK loop corrections on both the production and the decay of the Higgs boson. In order to take the different ratios of the production cross section into account, we employ the following quantity $[105,106]$ :

$$
\epsilon_{f}^{I, X}=\frac{a_{f}^{I, X} \sigma_{X}^{\mathrm{SM}}}{\sum_{Y} a_{f}^{I, Y} \sigma_{Y}^{\mathrm{SM}}},
$$

where $X$ and $I$ indicate a production channel and a category tagging the decay $H \rightarrow f, \sigma_{X}^{\mathrm{SM}}$ is the Higgs production cross section of the channel $X$ in the SM, and $a_{f}^{I, X}$ is introduced as its acceptance. When the set $\left\{\epsilon_{f}^{I, X}\right\}$ is given in the decay $H \rightarrow f$, the signal strength is written as

$$
\mu_{H \rightarrow f}^{I}=\sum_{X} \epsilon_{f}^{I, X} \frac{\sigma_{X}}{\sigma_{X}^{\mathrm{SM}}} \frac{\mathcal{B}_{H \rightarrow f}}{\mathcal{B}_{H \rightarrow f}^{\mathrm{SM}}},
$$

where $\sigma_{X}^{(\mathrm{SM})}$ represents the Higgs production cross section of the channel $X$, and $\mathcal{B}_{H \rightarrow f}^{(\mathrm{SM})}=\Gamma_{H \rightarrow f}^{(\mathrm{SM})} / \Gamma_{H \rightarrow \text { all }}^{(\mathrm{SM})}$ is the branching ratio of the Higgs decay $H \rightarrow f$ (in the SM). In the UED model, $\quad \sigma_{\mathrm{GF}}=\hat{\sigma}_{g g \rightarrow H}^{\mathrm{UED}}, \quad \Gamma_{H \rightarrow \gamma \gamma(g g)}=\Gamma_{H \rightarrow \gamma \gamma(g g)}^{\mathrm{UED}}$ as in Eqs. (16)-(18), and the others are assumed to be the same as the SM in our analysis.

For the analysis in $H \rightarrow \gamma \gamma$, the ATLAS and CMS experiments have shown their results of $\mu_{H \rightarrow \gamma \gamma}^{I}$ and the set $\left\{\epsilon_{\gamma \gamma}^{I, X}\right\}$ they used in their analyses [3,6]. We summarize these values in Tables III and IV. For the analysis in $H \rightarrow Z Z / W W$, the CMS result is summarized in Table V. The result of $H \rightarrow W W$ in the CMS experiment is given by assuming that all Higgs signals are produced by the GF process [8]. The ATLAS experiment only gives the signal strength for the specific production channels [4,5], which is written as 
TABLE IV. The CMS result of $H \rightarrow \gamma \gamma$ analysis. The CMS experiment defines these event categories and uses these ratios of the production channels as in Ref. [6].

\begin{tabular}{lccrrr}
\hline \hline$I$ & $\mu_{H \rightarrow \gamma \gamma}^{I}$ & \multicolumn{4}{c}{$\epsilon_{\gamma \gamma}^{I, X}(\%)$} \\
Event category & Signal strength & \multicolumn{1}{c}{$\mathrm{GF}$} & $\mathrm{VBF}$ & $\mathrm{VH}$ & $\mathrm{ttH}$ \\
\hline Missing $E_{T}$ & $1.9_{-2.3}^{+2.6}$ & 22.0 & 2.6 & 63.7 & 11.7 \\
Electron tag & $-0.7_{-2.0}^{+2.8}$ & 1.1 & 0.4 & 78.7 & 20.8 \\
muon tag & $0.4_{-1.4}^{+1.8}$ & \multicolumn{1}{c}{0} & 0.2 & 79.0 & 19.8 \\
2-jet loose & $0.8_{-1.0}^{+1.1}$ & 47.0 & 50.9 & 1.7 & 0.5 \\
2-jet tight & $0.3_{-0.6}^{+0.7}$ & 20.7 & 78.9 & 0.3 & 0.1 \\
Untag-3 & $-0.3_{-0.9}^{+0.8}$ & 92.5 & 3.9 & 3.3 & 0.3 \\
Untag-2 & $0.3 \pm 0.5$ & 91.6 & 4.5 & 3.6 & 0.4 \\
Untag-1 & $0.0 \pm 0.7$ & 83.5 & 8.4 & 7.1 & 1.0 \\
Untag-0 & $2.2_{-0.8}^{+0.9}$ & 72.9 & 11.6 & 12.9 & 2.6 \\
2-jet (7 TeV) & $4.2_{-1.8}^{+2.3}$ & 26.8 & 72.5 & 0.6 & 0 \\
Untag-3 (7 TeV) & $1.5_{-1.8}^{+1.7}$ & 91.3 & 4.4 & 4.1 & 0.2 \\
Untag-2 (7 TeV) & $0.0_{-1.2}^{+1.3}$ & 91.3 & 4.4 & 3.9 & 0.3 \\
Untag-1 (7 TeV) & $0.2_{-1.0}^{+1.0}$ & 87.6 & 6.2 & 5.6 & 0.5 \\
Untag-0 (7 TeV) & $3.8_{-1.7}^{+2.0}$ & 61.4 & 16.8 & 18.7 & 3.1 \\
\hline \hline
\end{tabular}

$$
\mu_{H \rightarrow Z Z / W W}^{X}=\frac{\sigma_{X}}{\sigma_{X}^{\mathrm{SM}}} \frac{\mathcal{B}_{H \rightarrow Z Z / W W}}{\mathcal{B}_{H \rightarrow Z Z / W W}^{\mathrm{SM}}} .
$$

The results are given as $\mu_{H \rightarrow Z Z}^{\mathrm{GF}+\mathrm{tH}}=1.8_{-0.5}^{+0.8}, \mu_{H \rightarrow Z Z}^{\mathrm{VBF}+\mathrm{VH}}=$ $1.2_{-1.4}^{+3.8}, \mu_{H \rightarrow W W}^{\mathrm{GF}}=0.82 \pm 0.36$, and $\mu_{H \rightarrow W W}^{\mathrm{VBF}}=1.66 \pm 0.79$. In this article, we assume $\mu_{H \rightarrow Z Z}^{\mathrm{GF}+\mathrm{ttH}} \simeq \mu_{H \rightarrow Z Z}^{\mathrm{GF}}$ for simplicity.

We evaluate a bound on the KK scale in each UED model by performing a $\chi^{2}$ analysis of the results as shown above. The $\chi^{2}$ function is represented as

$$
\chi^{2}=\sum_{f} \sum_{I}\left(\frac{\mu_{H \rightarrow f}^{I}-\hat{\mu}_{f}^{I}}{\hat{\sigma}_{f}^{I}}\right)^{2},
$$

where we assume the experimental results to be Gaussian distribution $\hat{\mu}_{f}^{I} \pm \hat{\sigma}_{f}^{I}$. ${ }^{6}$ The number of the observables we use in our analysis is 42 in total, and the degree of freedom is also the same number in terms of testing a justification of a model.

\section{Bound on KK scale from the current data}

Here, we show bounds on several UED models from the Higgs searches at the LHC. For our analyses, we have taken the highest possible UV cutoff scale $\Lambda_{\max }$ shown in Table II. The Higgs mass is chosen to be $126 \mathrm{GeV}$. In Fig. 5, we show the exclusion C.L. of each UED model as a function of the KK scale $M_{\mathrm{KK}}$ by use of all the ATLAS and CMS results of $H \rightarrow \gamma \gamma, W W, Z Z$. The black line indicates the result in the five-dimensional mUED model.

\footnotetext{
${ }^{6}$ Note that, since we neglect the correlation among the categories, which is not made public, this analysis should rather be taken as an illustration.
}

TABLE V. The CMS result of $H \rightarrow Z Z / W W$ analysis. The CMS experiment defines these event categories and uses these ratios of the production channels as in Refs. [7,8]. SF and DF denote "same flavor" and "different flavor," respectively.

\begin{tabular}{lccr}
\hline \hline$I$ & $\mu_{H \rightarrow Z Z}^{I}$ & \multicolumn{2}{c}{$\epsilon_{Z Z}^{I, X}(\%)$} \\
Event category & Signal strength & GF & VBF \\
\hline Untagged & $0.85_{-0.26}^{+0.32}$ & 95 & 5 \\
2-jet tag & $1.22_{-0.57}^{+0.84}$ & 80 & 20 \\
\hline \hline$I$ & $\mu_{H \rightarrow W W}^{I}$ & \multicolumn{2}{c}{$\epsilon_{W W}^{I, X}(\%)$} \\
\hline SF 1 jet (7 TeV) & $0.9_{-2.2}^{+2.1}$ & 100 & 0 \\
SF 0 jet (7 TeV) & $0.1 \pm 1.0$ & 100 & 0 \\
DF 1 jet (7 TeV) & $1.7 \pm 1.0$ & 100 & 0 \\
DF 0 jet (7 TeV) & $0.6 \pm 0.5$ & 100 & 0 \\
SF 1 jet (8 TeV) & $1.5 \pm 0.9$ & 100 & 0 \\
SF 0 jet (8 TeV) & $1.1 \pm 0.7$ & 100 & 0 \\
DF 1 jet (8 TeV) & $0.3 \pm 0.4$ & 100 & 0 \\
DF 0 jet (8 TeV) & $0.7 \pm 0.3$ & 100 & 0 \\
\hline \hline
\end{tabular}

The blue solid, dashed, and dotted lines denote those in the $T^{2}$-based ones, namely, the $T^{2} / Z_{2}, T^{2} /\left(Z_{2} \times Z_{2}^{\prime}\right)$, and $T^{2} / Z_{4}$, respectively. The red solid and dashed lines represent those in the $S^{2}$-based ones, namely, $S^{2}$ and $S^{2} / Z_{2}$, respectively. The green solid and dashed lines show those in the nonorientable ones, namely, $R P^{2}$ and PS, respectively.

As can be seen in this graph, we find that the region $M_{\mathrm{KK}} \lesssim 600 \mathrm{GeV}$ is excluded within $95 \%$ C.L. in the mUED model. For the six-dimensional models in the $T^{2}$-based space, we find the excluded regions $M_{\mathrm{KK}} \lesssim$ 1100,1000 , and $800 \mathrm{GeV}$ within $95 \%$ C.L. for $T^{2} / Z_{2}$, $T^{2} /\left(Z_{2} \times Z_{2}^{\prime}\right)$, and $T^{2} / Z_{4}$, respectively. For the $S^{2}$-based models, we can see that the regions $M_{\mathrm{KK}} \lesssim 1300$ and $900 \mathrm{GeV}$ are excluded within $95 \%$ C.L. in the $S^{2}$ and $S^{2} / Z_{2}$, respectively. For the nonoriented models, the regions $M_{\mathrm{KK}} \lesssim 1100$ and $1200 \mathrm{GeV}$ are excluded within

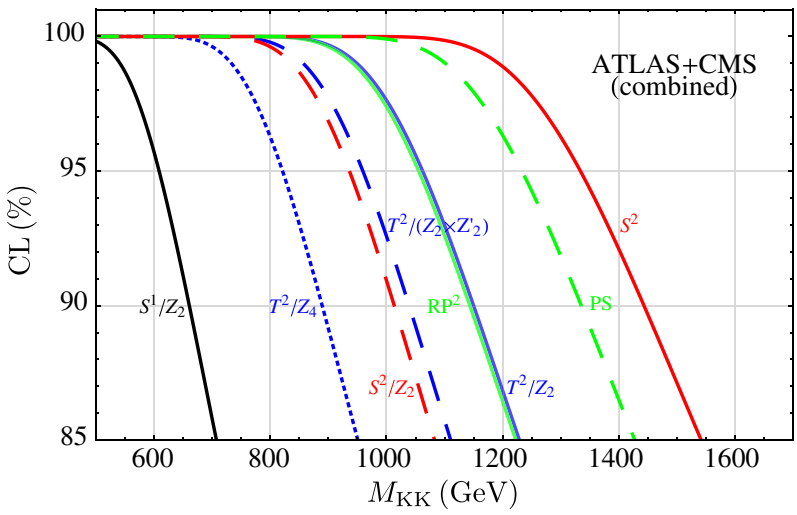

FIG. 5 (color online). Exclusion C.L.s of all the UED models as functions of the KK scale $M_{\mathrm{KK}}$ by use of all the ATLAS and CMS results of $H \rightarrow \gamma \gamma, W W, Z Z$. Colors denote the same as in Fig. 2. 

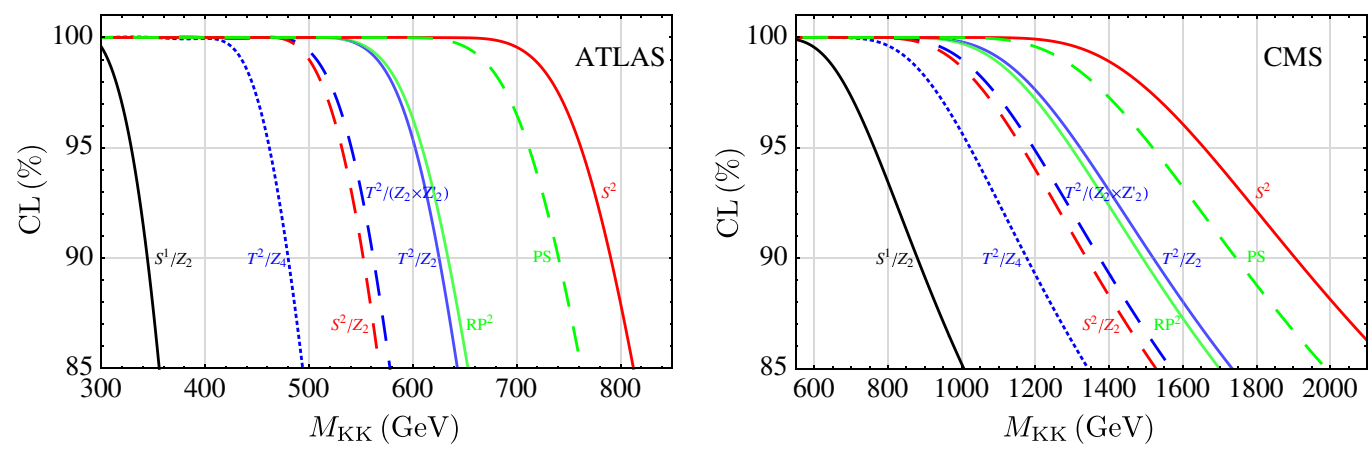

FIG. 6 (color online). The exclusion C.L.s of all UED models as functions of the KK scale $M_{\mathrm{KK}}$ obtained from the ATLAS (left) and CMS (right) results of $H \rightarrow \gamma \gamma, W W, Z Z$. Colors denote the same as in Fig. 2.

95\% C.L. in the $R P^{2}$ and PS, respectively. As seen above, the excluded region is different from one model to another in the case for UED. This is because the difference of the KK spectrum has a large impact on the Higgs decays via loop processes.

We compare the bounds obtained from the ATLAS experiment with those from the CMS in Fig. 6. We find that the CMS result gives a more stringent bound on the KK scale compared with the ATLAS one. In other words, for now, the UED models are likely to explain the recent ATLAS result, while they are disfavored by the recent CMS result. The results of the exclusion C.L.s for the wide range of the KK scale are summarized in Fig. 10 in Appendix D.

Throughout this analysis, we ignore the effects from the higher-dimensional operators around $\Lambda$. See Ref. [84] for such an effect.

\section{INDIRECT CONSTRAINT FROM $S$ AND $T$ PARAMETERS}

Physics beyond the SM is also restricted through the precise measurement of some electroweak variables. The $S$ and $T$ parameters proposed by Peskin and Takeuchi $[107,108]$ have been used for estimating whether a model is valid or not. The variables are defined by use of the two-point functions of the SM gauge bosons,

$$
\Pi_{a b}^{\mu \nu}(k)=i \Pi_{a b}^{\mathrm{T}}\left(k^{2}\right)\left(g^{\mu \nu}-\frac{k^{\mu} k^{\nu}}{k^{2}}\right)+i \Pi_{a b}^{\mathrm{L}}\left(k^{2}\right) \frac{k^{\mu} k^{\nu}}{k^{2}}
$$

where $k$ is the external momentum, T (L) means that it is the transverse (longitudinal) part, and the indices $a, b$ show types of the SM gauge bosons. The variables are constructed by the transverse components, and the concrete forms are written down with adapting the notation on the electroweak sector in Ref. [91] as

$$
\frac{\alpha S}{4 s_{W}^{2} c_{W}^{2}}=\Pi_{Z Z}^{\mathrm{T}}{ }^{\prime}(0)+\frac{c_{W}^{2}-s_{W}^{2}}{c_{W} s_{W}} \Pi_{Z \gamma}^{\mathrm{T}}{ }^{\prime}(0)-\Pi_{\gamma \gamma^{\prime}}^{\mathrm{T}}(0)
$$

$$
\alpha T=\frac{\Pi_{W W}^{\mathrm{T}}(0)}{m_{W}^{2}}-\frac{\Pi_{Z Z}^{\mathrm{T}}(0)}{m_{Z}^{2}}+2 c_{W} s_{W} \frac{\Pi_{Z \gamma}^{\mathrm{T}}(0)}{m_{W}^{2}},
$$

where $\Pi_{a b}^{\mathrm{T}}$ ' is defined as $\frac{d}{d k^{2}} \Pi_{a b}^{\mathrm{T}}\left(k^{2}\right)$.

The $S$ and $T$ are also described by combinations of some electroweak variables, and their values are calculated in global analysis with experimental results. One of the latest numbers is found in Ref. [109],

$\left.S\right|_{U=0}=0.05 \pm 0.09,\left.\quad T\right|_{U=0}=0.08 \pm 0.07, \quad \rho_{S T}=+0.91$,

with $126 \mathrm{GeV}$ reference Higgs mass and assuming the $U$ parameter is zero and $\rho_{S T}$ is the correlation coefficient. In an operator-analysis point of view, the $U$ parameter is represented as a coefficient of a much higher-dimensional operator with the Higgs doublet compared with $S$ and $T$ in the UED models, and hence we ignore the effect in our analysis.

\section{A. Forms in 6D UED models and mUED}

In this section, we formulate the contributions to the $S$ and $T$ parameters in the 6D UED models and in the mUED model. It is well known that the $S$ and $T$ parameters are logarithmically divergent in six dimensions [88]. To have a rough idea of what happens, we employ the following prescription. First, we compute the contributions from each KK mode within four-dimensional field theory employing the dimensional regularization. They are manifestly finite. Then, we sum such contributions up to a mode in which the KK mass exceeds the UV cutoff $\Lambda$. To estimate the possible effects from the UV theory above $\Lambda$, we also put the higher-dimensional operators in six dimensions.

The general shape of the $S$ and $T$ parameters are

$S=\sum_{\substack{s \\ \text { with } M_{s}<\Lambda}}\left(S_{s, \text { boson }}^{(\mathrm{KK})}+S_{s, \text { fermion }}^{(\mathrm{KK})}\right)+S_{\text {Higgs calibration }}+S_{\text {threshold }}$ 


$$
T=\sum_{\substack{s \\ \text { with } M_{s}<\Lambda}}\left(T_{s, \text { boson }}^{(\mathrm{KK})}+T_{s, \text { fermion }}^{(\mathrm{KK})}\right)+T_{\text {Higgs calibration }}+T_{\text {threshold }} \text {, }
$$

where the first terms in parentheses are the contributions of KK particles, and the last two terms represent, respectively, the effects from Higgs mass calibration and the threshold correction via possible higher-dimensional operators around the UV cutoff scale $\Lambda$ in six dimensions. These effects were considered in Refs. [88,108],

$$
\begin{gathered}
S_{\text {Higgs calibration }}=\frac{1}{12 \pi} \ln \left(\frac{m_{H}^{2}}{m_{H, \text { ref }}^{2}}\right), \\
T_{\text {Higgs calibration }}=-\frac{3}{12 \pi c_{W}^{2}} \ln \left(\frac{m_{H}^{2}}{m_{H, \text { ref }}^{2}}\right), \\
S_{\text {threshold }}=c_{S} \frac{2 \pi v^{2}}{\Lambda^{2}}, \quad T_{\text {threshold }}=c_{T} \frac{m_{H}^{2}}{4 \alpha \Lambda^{2}},
\end{gathered}
$$

where $m_{H \text {,ref }}$ is the assumed SM Higgs mass in global analysis, and $c_{S}$ and $c_{T}$ are undetermined dimensionless coefficients with $\mathcal{O}(1)$ magnitude.

Several comments are in order. One is that the summations over KK states are truncated at the scale $\Lambda$. The other is that the value of $\Lambda$ is estimated through the vacuum stability condition of the Higgs boson. We choose the highest possible $\Lambda$ allowed by it. As we discussed in Sec. II, in the configuration of $m_{H}=126 \mathrm{GeV}$, the value of the maximum UV cutoff scale tends to be low, and the threshold corrections possibly become important. We will include these effects below. Finally, we comment on the contributions of KK particles. We find that the effect from the state-s fermion loops takes the following general shapes in every 6D UED model, which is the same as in the mUED and was already calculated in Ref. [88]. We show them in our notation:

$S_{\text {fermion }, s}^{(\mathrm{KK})} \simeq \frac{1}{4 \pi} \frac{2}{3} x_{t, s}, \quad T_{\text {fermion }, s}^{(\mathrm{KK})} \simeq \frac{1}{\alpha}\left(\frac{m_{t}^{2}}{4 \pi^{2} v^{2}}\right) x_{t, s}$,

where $x_{t, s}$ is defined with the KK mass of the state " $s$ " $M_{s}$ as

$$
x_{t, s}=\frac{m_{t}^{2}}{M_{s}^{2}}
$$

and we ignore their $\mathcal{O}\left(x_{t, s}^{2}\right)$ corrections. In the $R P^{2}$ model, we should pay attention to the fact that the summation range differs between bosonic and fermionic sectors.

The bosonic part is highly model dependent. In this paper, we have newly calculated the contributions to $S$ and $T$ in every $6 \mathrm{D}$ model. The complete forms of the gauge-boson two-point functions are summarized in Appendix C.

In the cases of $T^{2} / Z_{2}, T^{2} /\left(Z_{2} \times Z_{2}^{\prime}\right), T^{2} / Z_{4}, S^{2}$, and $S^{2} / Z_{2}$, the forms are

$$
\begin{aligned}
S_{\text {boson }, s}^{(\mathrm{KK})} \simeq \frac{1}{\pi}\{ & \left.-\frac{5}{36} x_{W, s}+\frac{1}{24} x_{H, s}+\left(\frac{1}{24}-\frac{1}{6 c_{W}^{2}}\right) x_{Z, s}\right\}, \\
T_{\text {boson }, s}^{(\mathrm{KK})} \simeq & \frac{1}{4 \pi} \frac{1}{s_{W}^{2}}\left\{\left(\frac{15}{4}-\frac{193}{72} \frac{1}{c_{W}^{2}}+\frac{1}{2 c_{W}^{4}}\right) x_{W, s}\right. \\
& +\left(-\frac{85}{72}-\frac{7}{18} \frac{1}{c_{W}^{2}}\right) x_{Z, s} \\
& \left.+\left(\frac{13}{36}+\frac{5}{36} \frac{1}{c_{W}^{2}}-\frac{1}{2 c_{W}^{4}}\right) x_{H, s}\right\},
\end{aligned}
$$

where we define similar variables as in Eq. (32): $x_{i, s}=\frac{m_{i}^{2}}{M_{s}^{2}}$ with $m_{W}^{2}, m_{Z}^{2}$, and $m_{H}^{2}$. Note that the lighter the $\mathrm{KK}$ particles are the greater they contribute to $S$ and $T$. In these models, the result is affected only by the differences in the patterns of the surviving KK modes.

In the cases of the models based on the nonorientable manifolds $R P^{2}$ and PS, bosonic contributions are classified into three and two categories, respectively. The details of the following classifications have already been discussed in Sec. II, and thus we do not explain it here. The results in the PS model are shown:

$$
\begin{aligned}
& S_{\text {boson, }, \text { :odd }}^{(\mathrm{KK})} \simeq 0, \\
& T_{\text {boson, } s: \text { odd }}^{(\mathrm{KK})} \simeq \frac{1}{4 \pi} \frac{1}{s_{W}^{2}} \frac{5}{18}\left\{\left(1-\frac{1}{c_{W}^{2}}\right) x_{W, s}+\left(\frac{1}{c_{W}^{2}}-1\right) x_{Z, s}\right\}, \\
& S_{\mathrm{boson}, s \text { even }}^{(\mathrm{KK})} \simeq \frac{1}{\pi}\left\{-\frac{5}{36} x_{W, s}+\frac{1}{24} x_{H, s}+\left(\frac{1}{24}-\frac{1}{6 c_{W}^{2}}\right) x_{Z, s}\right\}, \\
& T_{\text {boson, } s: \text { even }}^{(\mathrm{KK})} \simeq \frac{1}{4 \pi} \frac{1}{s_{W}^{2}}\left\{\left(\frac{125}{36}-\frac{173}{72} \frac{1}{c_{W}^{2}}+\frac{1}{2 c_{W}^{4}}\right) x_{W, s}\right. \\
& +\left(-\frac{65}{72}-\frac{2}{3} \frac{1}{c_{W}^{2}}\right) x_{Z, s} \\
& \left.+\left(\frac{13}{36}+\frac{5}{36} \frac{1}{c_{W}^{2}}-\frac{1}{2 c_{W}^{4}}\right) x_{H, s}\right\} \text {. }
\end{aligned}
$$

The shapes in the $R P^{2}$ model are closely related the previous ones in the PS as follows:

$$
\begin{gathered}
\{S, T\}_{\text {boson, } s: \text { region I }}^{(\mathrm{KK})}=\{S, T\}_{\text {boson, } s: \text { even }}^{(\mathrm{KK})} \\
\{S, T\}_{\text {boson, } s: \text { region II }}^{(\mathrm{KK})}=\{S, T\}_{\text {boson, } s: \text { odd }}^{(\mathrm{KK})}, \\
\{S, T\}_{\text {boson, } s: \text { region III }}^{(\mathrm{KK})}=\{S, T\}_{\text {boson, },}^{(\mathrm{KK})}
\end{gathered}
$$

where we note that we should use the form of the KK mass on $S^{2}$ instead of on $T^{2}$.

The mixing among KK states in the gauge sector is schematically of the form 


$$
\left[\begin{array}{ccc}
m_{W, Z}^{2}+M_{\mathrm{KK}}^{2} & M_{\mathrm{KK}}^{2} & m_{W, Z} M_{\mathrm{KK}} \\
M_{\mathrm{KK}}^{2} & m_{W, Z}^{2}+M_{\mathrm{KK}}^{2} & m_{W, Z} M_{\mathrm{KK}} \\
m_{W, Z} M_{\mathrm{KK}} & m_{W, Z} M_{\mathrm{KK}} & m_{W, Z}^{2}+M_{\mathrm{KK}}^{2}
\end{array}\right] .
$$

In the calculation of $S$ and $T$ parameters, we adopt the following approximation about the mass mixings of $6 \mathrm{D} \mathrm{W}$ and $\mathrm{Z}$ boson-related sectors:

(i) We ignore off-diagonal terms with the magnitude $\mathcal{O}\left(m_{W, Z} M_{\mathrm{KK}}\right)$, which are small compared with the other terms with the magnitude $\mathcal{O}\left(M_{\mathrm{KK}}^{2}\right)$.

(ii) In the diagonal terms, for which the forms are approximately as $m_{W, Z}^{2}+M_{\mathrm{KK}}^{2}$, we do not ignore the small part coming from $m_{W, Z}^{2}$ since this part can contribute to the $T$ parameter.

Because of this approximation, the small mixings being proportional to $m_{W, Z}^{2}$ are ignored. As a result, some divergent terms that are proportional to $m_{W, Z}^{2}$ remain in the $T$ parameter, and we simply discard them. Note that the contribution to $T$ from each $\mathrm{KK}$ mode must be manifestly finite since it is computed in four-dimensional field theory with the dimensional regularization. The divergence $\left(\propto m_{W, Z}^{2}\right)$ that we encounter here is an artifact coming from the ignorance of the small off-diagonal part in the KK mixing of the gauge sector. Indeed, we find that there appears no divergence proportional to $m_{H}^{2}$ or $m_{t}^{2}$, as we treat the mixing of the Higgs and top KK sectors exactly. Although there might be a further possible finite correction due to this procedure, the $\mathrm{KK}$ gauge contribution is generally subleading compared to the KK top loops, the mixing of which we treat exactly. In the $S$ parameter, we do not see any divergence even after the above approximation. These features are consistent with the general property of $S$ and $T$.

After we considered radiative corrections, the Weinberg angles of the $\mathrm{KK} W$ and $Z$ bosons get to be very small [110]. We assume in this effect that the KK Weinberg angles are zero and that we can simply ignore the mass corrections. Each KK-state contribution should be suppressed by its KK mass, and hence this effect should not affect the leading order of $S$ and $T$ since their contributions are proportional to $\mathrm{KK}$ masses (when we ignore the electroweak masses in loop calculation) [110].

Finally we comment on the mUED model, which has been studied extensively [18,22,88,111,112]. In the $\chi^{2}$ analysis of Refs. [22,88], the authors simply ignored the terms being proportional to $m_{W, Z}^{2}$, possibly because their effects are not significant compared with those that are proportional to $m_{H}^{2}$ or $m_{t}^{2}$.

Boson contributions to $S$ and $T$ in the mUED are approximately described with the even part of the PS (or region I of the $R P^{2}$ ) as we already showed in Eqs. (36) and (37) since the particle content of each KK state is the same. Fermion contributions are the same as in Eq. (31). Here, we adopt the form of the KK mass $M_{(n)}$ with a KK number $n$

$$
M_{(n)}^{2}=n^{2} M_{\mathrm{KK}}^{2}, \quad(\text { for } n=1,2,3, \cdots) .
$$

\section{B. Numerical results without threshold correction}

We also execute a $\chi^{2}$ analysis for putting indirect constraints on the UED models. $\chi^{2}$ from $S$ and $T$ is defined as

$$
\begin{aligned}
\chi_{S T}^{2}= & \frac{1}{1-\rho_{S T}^{2}}\left\{\frac{\left(S-S_{\exp }\right)^{2}}{\sigma_{S}^{2}}+\frac{\left(T-T_{\exp }\right)^{2}}{\sigma_{T}^{2}}\right. \\
& \left.-\frac{2 \rho_{S T}}{\sigma_{S} \sigma_{T}}\left(S-S_{\exp }\right)\left(T-T_{\exp }\right)\right\},
\end{aligned}
$$

where $S$ and $T$ are the theoretical inputs in Eqs. (27) and (28), and the others are the experimental resultants in Eq. (26). In this and the next sections, we again adopt the assumption of $R_{5}=R_{6}=R$.

At first in this section, we consider the possibility without threshold correction to $S$ and $T$ in Eq. (30). We consider the maximal cutoffs with $M_{\mathrm{KK}}=1 \mathrm{TeV}$ irrespective of $M_{\mathrm{KK}}$ because our interest is in the case that $M_{\mathrm{KK}}$ is about a few $\mathrm{TeV}$, and the values are almost universal as a function of $M_{\mathrm{KK}}$ in each model around a few TeV as shown in Fig. 2. The result is listed in Fig. 7. The plots for a wider range of $M_{\mathrm{KK}}$ are shown in Appendix D, where we can find the global minima in every curve. Each minimum is located around $1700 \mathrm{GeV}\left(T^{2} / Z_{2}\right), 1500 \mathrm{GeV}\left(T^{2} /\left(Z_{2} \times Z_{2}^{\prime}\right)\right)$, $1300 \mathrm{GeV}\left(T^{2} / Z_{4}\right), 2200 \mathrm{GeV}\left(S^{2}\right), 1500 \mathrm{GeV}\left(S^{2} / Z_{2}\right)$, $1800 \mathrm{GeV}\left(R P^{2}\right), 2000 \mathrm{GeV}$ (PS), and $1000 \mathrm{GeV}$ (mUED). Interestingly, these values are somewhat greater than the corresponding $95 \%$ C.L. bound from the combined results in the Higgs searches as shown in Fig. 5.

We also estimate the $95 \%$ C.L. bounds of the models from Fig. 7, and the values are about $1200 \mathrm{GeV}\left(T^{2} / Z_{2}\right)$, $1100 \mathrm{GeV}\left(T^{2} /\left(Z_{2} \times Z_{2}^{\prime}\right)\right), 900 \mathrm{GeV}\left(T^{2} / Z_{4}\right), 1500 \mathrm{GeV}$ $\left(S^{2}\right), 1100 \mathrm{GeV}\left(S^{2} / Z_{2}\right), 1200 \mathrm{GeV}\left(R P^{2}\right), 1400 \mathrm{GeV}(\mathrm{PS})$, and $700 \mathrm{GeV}$ (mUED). Here, we can notice that these indirect bounds are compatible with the direct bounds via the $\mathrm{LHC}$ results discussed in the previous section. We note

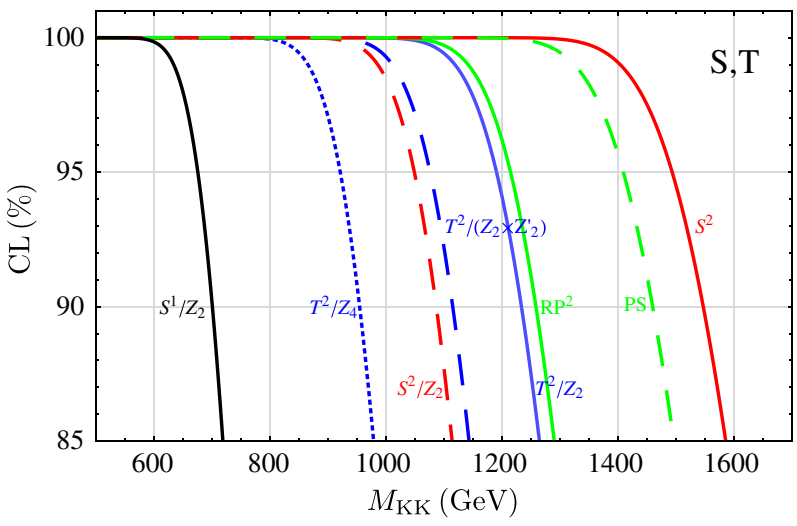

FIG. 7 (color online). The exclusion C.L.s of all the UED models as functions of $M_{\mathrm{KK}}$ obtained from the $S$ and $T$ parameters. Colors denote the same as in Fig. 2. 

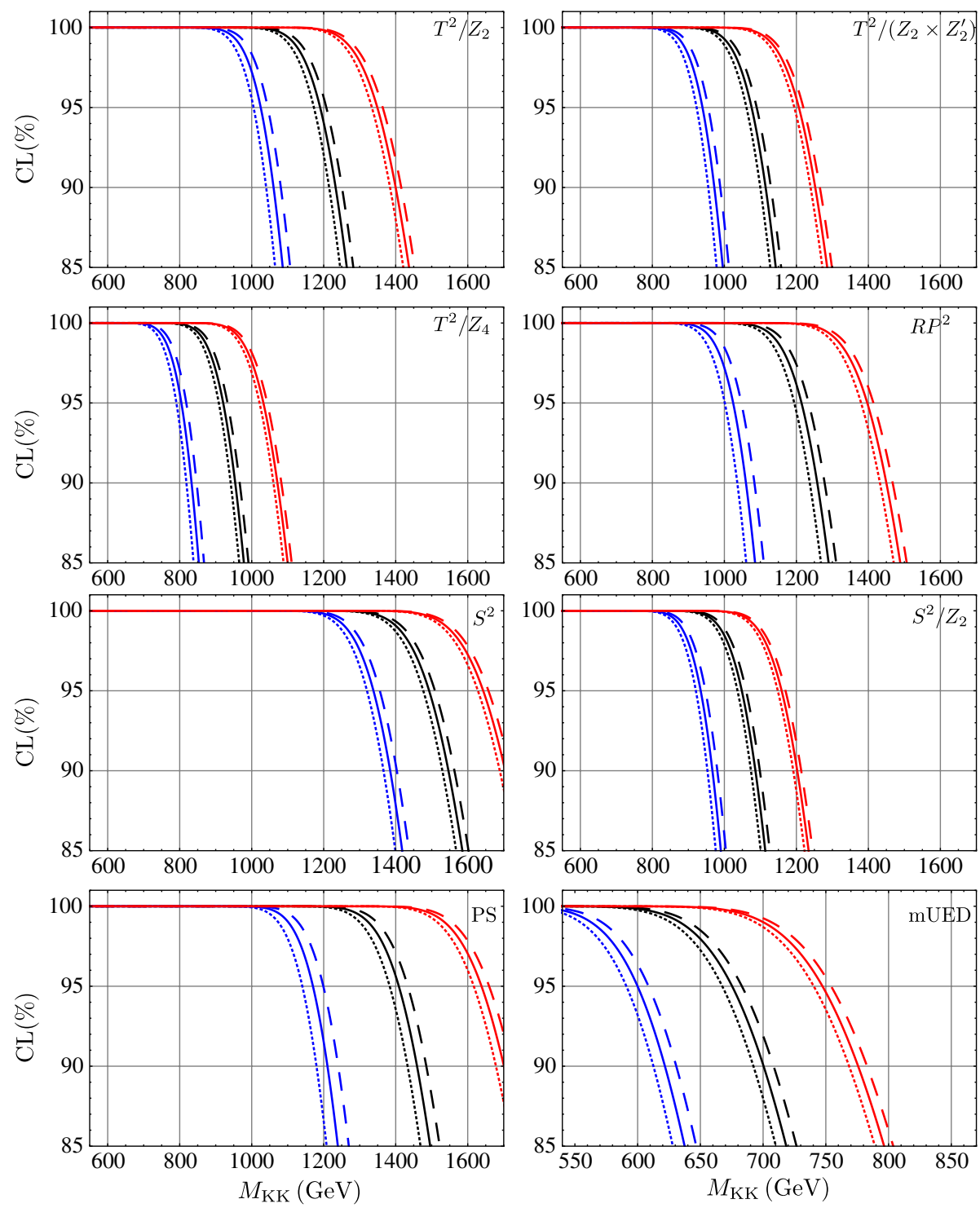

FIG. 8 (color online). The exclusion C.L.s via the $\chi^{2}$ analysis of $S$ and $T$ parameters as a function of $M_{\mathrm{KK}}$ with the maximal cutoffs in the UED models in Table II with threshold corrections in Eq. (30). In each panel corresponding to each model, the left (blue), center (black), and right (red) bunches of lines are for $c_{S}=+1,0$, and -1 , respectively. In each bunch, the dotted, solid, and dashed lines correspond to $c_{T}=-1,0$, and +1 , respectively.

that our $95 \%$ C.L. bound ( $700 \mathrm{GeV}$ ) on the mUED is close to the previous values by the Gfitter group $(700 \mathrm{GeV}$ in $m_{H}=126 \mathrm{GeV}$ ) in Ref. [22].

\section{Numerical results with threshold correction}

As we have seen in Sec. II, the vacuum is destabilized rapidly for the Higgs mass $m_{H}=126 \mathrm{GeV}$, and we should take the cutoff scale quite a low. For completeness, we estimate the threshold corrections via physics around the cutoff scale $\Lambda$.

In this section, we summarize the results, for the maximum UV cutoff scale, given in Table II, in Fig. 8. Here, we examine the three extremal possibilities $\left(c_{S, T}=0,+1,-1\right)$ for each of the two coefficients $c_{S}$ and $c_{T}$ in Eq. (30).
We can find sizable deviations from the case of $c_{S}=$ $c_{T}=0$ in all the models. The corrections from $S_{\text {threshold }}$ are significant, and the $95 \%$ C.L. bounds turn out to be modified by the magnitudes about $100-200 \mathrm{GeV}$, depending on the models, toward both positive and negative directions, while the corrections from $T_{\text {threshold }}$ are subleading. We note that we can find the global minimum in all the models after taking into account the threshold corrections, which is not shown in Fig. 8.

One important thing is that, even in the 5D mUED, the threshold corrections are more significant than was thought. We report that the $T^{2} / Z_{2}$ case was studied in Ref. [88] with $\Lambda=5 M_{\mathrm{KK}}$. In our result, the degree of the deviations from the case without the threshold correction is enlarged since we can take $\Lambda$ at most $2.5 M_{\mathrm{KK}}$ as shown in Table II. 


\section{SUMMARY AND DISCUSSIONS}

We have studied the effects from the KK particles in the UED models on the Higgs searches at the LHC and on the electroweak precision data. Both are dependent on the UV cutoff scale $\Lambda$ of the higher-dimensional theory. We have evaluated the highest possible $\Lambda$ consistent with the vacuum stability bound on the Higgs potential.

In the UED models, the contributions from loop diagrams including the $\mathrm{KK}$ top quarks and gauge bosons modify the Higgs decay rate and production cross section, which affect the Higgs signal strengths at the LHC. On the other hand, the KK excited states of the heavier SM particles (top quark, Higgs boson, and the massive gauge bosons) alter the $S$ and $T$ parameters. From the analysis on the results of the Higgs signal strengths in the decay modes $H \rightarrow \gamma \gamma, Z Z, W W$ and of the $S$ and $T$ parameters, we have estimated the two types of bounds on the KK scales in 5D and 6D UED models, which are summarized in Table VI. Comparing the former bounds with the latter, we find that the latter are slightly more severe than the former. However, in few years, the Higgs searches at the LHC will put stronger constraints on the KK scale in the UED models. We note that there remain uncertainties from the choices of the UV cutoff $\Lambda<\Lambda_{\max }$, the higherdimensional operators there, and the low-energy input for the top Yukawa coupling. In the estimation of $\Lambda_{\max }$, we focus on the vacuum stability bound, namely, the condition (13) on the coupling $\lambda$. This new bound is tighter than the conventional one derived from the perturbativity of the gauge couplings. It might be interesting to take into account the effects of higher-dimensional operators for the stability argument, as we have done for the $S$ and $T$, since the scale of $\Lambda_{\max }$ tends to be low in the UED models after the Higgs discovery.

The authors of Ref. [113] have shown that the case $R_{5}=$ $R_{6}$ is disfavored in the $R P^{2}$ model if we identify the lowest KK state to be the dark matter, compared with the limit $R_{5} \gg R_{6}$, because the former requires lower dark matter mass than the latter. We show in Fig. 9 the same exclusion limits from the Higgs searches and from $S, T$, as given above, in this five-dimensional limit. As expected, the

TABLE VI. Highest possible UV cutoff scales and lower bounds on the KK scale $M_{\mathrm{KK}}$ for each model at the $95 \%$ C.L.

\begin{tabular}{lccc}
\hline \hline & $\Lambda / M_{\mathrm{KK}}$ for & Higgs signal & $S$ and $T$ \\
& $M_{\mathrm{KK}} \sim O(\mathrm{TeV})$ & $\begin{array}{c}\text { strength }(\mathrm{GeV}) \\
\text { serameters }(\mathrm{GeV})\end{array}$ \\
\hline $\mathrm{mUED}$ & 5.0 & 610 & 680 \\
$T^{2} / Z_{2}$ & 2.5 & 1060 & 1190 \\
$T^{2} /\left(Z_{2} \times Z_{2}^{\prime}\right)$ & 2.9 & 960 & 1080 \\
$T^{2} / Z_{4}$ & 3.4 & 820 & 920 \\
$R P^{2}$ & 2.3 & 1060 & 1220 \\
$S^{2}$ & 2.3 & 1330 & 1490 \\
$S^{2} / Z_{2}$ & 3.2 & 940 & 1050 \\
$\mathrm{PS}$ & 1.9 & 1240 & 1410 \\
\hline \hline
\end{tabular}

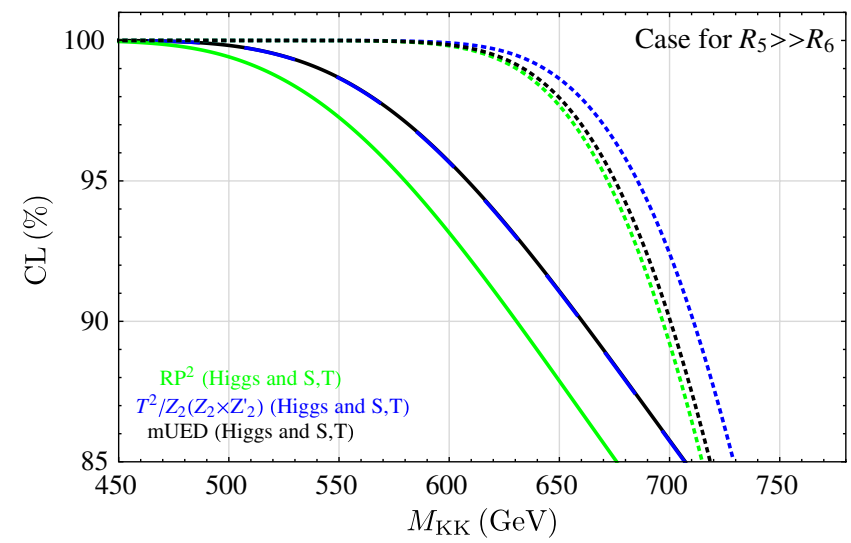

FIG. 9 (color online). The same exclusion C.L.s as above, in the limit $R_{5} \gg R_{6}$. The solid and dotted lines correspond to the bounds from Higgs searches and $S, T$ constraints, respectively. The former Higgs bounds for $T^{2} / Z_{2}\left(Z_{2} \times Z_{2}^{\prime}\right)$ and mUED are degenerate with each other.

bounds become almost the same as those in the mUED model, expect for the small difference due to extra contributions from the $6 \mathrm{D} \mathrm{KK}$ states of the gauge field. Note also that the five-dimensional limit $R_{5} \gg R_{6}$ loosens the vacuum stability bound and hence allows the higher cutoff scale $\Lambda_{\max }=5.5 M_{\mathrm{KK}}$ and $3.9 M_{\mathrm{KK}}$ for $T_{2} / Z_{2}\left(Z_{2} \times Z_{2}^{\prime}\right)$ and $R P^{2}$, respectively. The higher the cutoff scale is the larger the number of KK states below it. We have chosen $\Lambda=\Lambda_{\max }$ and taken into account this effect in Fig. 9.

We briefly comment on other bounds. We can find the recent studies in bounds from collider simulations in the mUED, 6D UEDs on $T^{2} / Z_{4}$, and $R P^{2}$.

(i) On mUED in Ref. [42]: $M_{\mathrm{KK}} \gtrsim 1300 \mathrm{GeV}$ with 95\% C.L. through trilepton signature + Missing ET (MET) with $20 \mathrm{fb}^{-1}$ at $\sqrt{s}=8 \mathrm{TeV}(\Lambda R=10)$.

(ii) On $T^{2} / Z_{4}$ in Ref [64]: $M_{\mathrm{KK}} \gtrsim 500 \mathrm{GeV}$ with $5 \sigma$ C.L. through $n$-jets $+\gamma+\operatorname{MET}(n \geq 4)$ with $2 \mathrm{fb}^{-1}$ at $\sqrt{s}=7 \mathrm{TeV}$.

(iii) On $R P^{2}$ in Ref. [68]: $M_{\mathrm{KK}} \gtrsim 600 \mathrm{GeV}$ with above 99\% C.L. through CMS $\alpha_{T}$ analysis in leptons + MET with $\sim 5 \mathrm{fb}^{-1}$ at $\sqrt{s}=7 \mathrm{TeV}$.

The constraint on the mUED is tighter than ours from the direct Higgs search and $S, T$ parameters, while these on $T^{2} / Z_{4}$ and $R P^{2}$ (with the limited integrated luminosities) are somewhat loose compared with ours. As pointed out in Ref. [100], a UED model with a low cutoff scale results in a much compressed KK spectrum and hence becomes difficult to detect at the LHC. It is noted that such a degenerate possibility has not been explored enough, and the analysis with $M_{T 2}$ and/or event shape variables is suitable for the case $[39,40]$. We also refer to the bounds from dark matter relic abundance in these three models. The upper bound on $M_{\mathrm{KK}}$ is approximately less than $200 \mathrm{GeV}$ in $T^{2} / Z_{4}$ [114] and $470 \mathrm{GeV}$ in $R P^{2}$ with $R_{5}=$ $R_{6}$ [113], to circumvent an overabundance of matter in the Universe. This bound suggests that these 6D UEDs on both 
TABLE VII. Prediction on the UED/SM ratio of $\mathrm{BR}(H \rightarrow \gamma \gamma)$ and $\sigma_{\gamma \gamma \rightarrow H}$ with the lowest possible value of the KK scale.

\begin{tabular}{lcc}
\hline \hline & $\begin{array}{c}\text { UED/SM ratio of } \\
\mathrm{BR}(H \rightarrow \gamma \gamma)\end{array}$ & $\begin{array}{c}\text { UED/SM ratio } \\
\text { of } \sigma_{\gamma \gamma \rightarrow H}\end{array}$ \\
\hline mUED & 0.93 & 0.94 \\
$T^{2} / Z_{2}$ & 0.93 & 0.94 \\
$T^{2} /\left(Z_{2} \times Z_{2}^{\prime}\right)$ & 0.93 & 0.94 \\
$T^{2} / Z_{4}$ & 0.92 & 0.94 \\
$R P^{2}$ & 0.93 & 0.94 \\
$S^{2}$ & 0.85 & 0.88 \\
$S^{2} / Z_{2}$ & 0.92 & 0.94 \\
$\mathrm{PS}$ & 0.90 & 0.92 \\
\hline \hline
\end{tabular}

geometries are disfavored in combination with our results. ${ }^{7}$ In the mUED, the range being consistent with the relics is $1300 \mathrm{GeV} \lesssim M_{\mathrm{KK}} \lesssim 1500 \mathrm{GeV}$ [21], which is just an unexplored area.

We have studied the suppression effects of Higgs decay into a diphoton in Sec. III. These effects can also affect the measuring of the Higgs to diphoton coupling at a future linear collider [115]. We summarize in Table VII the ratio of $\mathrm{BR}(H \rightarrow \gamma \gamma)$ as well as the Higgs production cross section from $\gamma \gamma$ collision in each UED model to those in the SM for the lowest possible KK scale with the highest possible UV cutoff. We find that the branching ratio and the Higgs production can be suppressed by a factor $\sim 0.9$ compared with SM. This is marginally accessible at the linear collider with integrated luminosity $500 \mathrm{fb}^{-1}$ at $500 \mathrm{GeV}$ for which the expected precision for the $\operatorname{BR}(H \rightarrow \gamma \gamma)$ is $23 \%$ for $M_{h}=120 \mathrm{GeV}$ [116]. This precision is refined to $5.4 \%$ with luminosity $1 \mathrm{ab}^{-1}$ at $1 \mathrm{TeV}$ for the same Higgs mass [117]. When we employ the photon-photon collider option, $H \gamma \gamma$ coupling can be measured more directly from the total production cross section of the Higgs. This is well within the reach for an integrated photon-photon luminosity $410 \mathrm{fb}^{-1}$ at a linear $e^{+} e^{-}$collider operated at a $\sqrt{s}=$ $210 \mathrm{GeV}$, which can measure $\Gamma_{H \rightarrow \gamma \gamma} \times \mathrm{BR}(H \rightarrow \gamma \gamma)$ with an accuracy of $2.1 \%$ for $m_{H}=120 \mathrm{GeV}$ [118].

\section{ACKNOWLEDGMENTS}

We thank Tomohiro Abe for useful comments on oblique corrections and Swarup Kumar Majee for discussions in the early stages of this work. K. N. is grateful for valuable discussions with Joydeep Chakrabortty and Daisuke Harada and for fruitful conversations with Anindya Datta and Sreerup Raychaudhuri. K. N. is partially supported by funding available from the Department of Atomic Energy, Government of India for the Regional Centre for Accelerator-based Particle Physics (RECAPP), Harish-Chandra Research Institute. The work

\footnotetext{
${ }^{7}$ In the $R P^{2}$ case, the upper bound on $M_{\mathrm{KK}}$ can be uplifted around $1.5 \mathrm{TeV}$ by introducing tuning in the parameter space $[68,113]$.
}

of K. O. (R.W.) is in part supported by the Grands-in-Aid for Scientific Research No. 23104009, No. 20244028, and No. 23740192 (No. 248920).

\section{APPENDIX A: RGES IN 6D UED MODELS AND mUED}

In this appendix, we show the concrete forms of RGEs for gauge, Yukawa, and Higgs self-couplings. Here, we rewrite the schematic shape of the beta function for a quantity $\mathcal{Q}$ in $6 \mathrm{D}$ UED:

$$
\beta_{Q}=\beta_{Q}^{(\mathrm{SM})}+\sum_{s: \text { KK states }} \theta\left(\mu-M_{s}\right)\left(N_{s} \beta_{s, Q}^{(\mathrm{KK})}\right) .
$$

Details of this expression are found in Sec. II A. As we have already discussed there, the beta functions take different forms depending on the following two categories: UEDs on an orientable space and those on an unorientable one. The former contains $T^{2} / Z_{2}, T^{2} /\left(Z_{2} \times Z_{2}^{\prime}\right), T^{2} / Z_{4}, S^{2}$, and $S^{2} / Z_{2}$ and the latter the remains $R P^{2}$, PS. The RGEs obtained in this work are consistent with those obtained for mUED [97] and for the SM [119]. The contribution of the $\mathrm{KK}$ particles to the beta function $\beta_{s, 2}^{(\mathrm{KK})}$ is independent of the KK index, and we can omit the index $s$ as $\beta_{Q}^{(\mathrm{KK})}$. We already explained the reason in Sec. II A. We note that in all the RGE analyses in this paper, we ignore Yukawa couplings except for the top quark one.

\section{UEDs in orientable space}

In the following, $\lambda$ is 4D Higgs self-couplings; $g_{1}, g_{2}$, and $g_{3}$ show the $4 \mathrm{D} U(1)_{Y}, S U(2)_{W}$, and $S U(3)_{C}$ gauge couplings; and $y_{\ell_{k}}, y_{u_{k}}, y_{d_{k}}(k=1,2,3)$ represent the $4 \mathrm{D}$ (diagonalized) Yukawa couplings of the charged leptons, the up-type quarks, and the down-type quarks, respectively. Here, we adopt the SM normalization in the $U(1)_{Y}$ gauge coupling $g_{1}$. The index $k$ indicates their generations. $V_{i j}$ means the Cabibbo-Kobayashi-Maskawa matrix, and $N_{C_{f_{i}}}$ indicates the color factor of the particle $f_{i}$, namely, 3 for quarks and 1 for leptons,

(i) $\mathcal{Q}=\lambda$

$$
\begin{aligned}
\beta_{\lambda}^{(\mathrm{SM})}= & \frac{1}{(4 \pi)^{2}}\left\{6 \lambda^{2}-\left(3 g_{1}^{2}+9 g_{2}^{2}\right) \lambda\right. \\
& +\frac{3}{2}\left(g_{1}^{4}+2 g_{1}^{2} g_{2}^{2}+3 g_{2}^{4}\right) \\
& \left.+4 \lambda \sum_{i} N_{C_{f_{i}}} y_{f_{i}}^{2}-8 \sum_{i} N_{C_{f_{i}}} y_{f_{i}}^{4}\right\} \\
\beta_{\lambda}^{(\mathrm{KK})}= & \frac{1}{(4 \pi)^{2}}\left\{6 \lambda^{2}-\left(3 g_{1}^{2}+9 g_{2}^{2}\right) \lambda\right. \\
& +\left(\frac{5}{2} g_{1}^{4}+5 g_{1}^{2} g_{2}^{2}+\frac{15}{2} g_{2}^{4}\right) \\
& \left.+8 \lambda \sum_{i} N_{C_{f_{i}}} y_{f_{i}}^{2}-16 \sum_{i} N_{C_{f_{i}}} y_{f_{i}}^{4}\right\} .
\end{aligned}
$$


(ii) $\mathcal{Q}=g_{i}(i=1,2,3)$

$$
\begin{aligned}
& \beta_{g_{i}}^{(\mathrm{SM})}=\frac{1}{(4 \pi)^{2}} b_{g_{i}}^{(\mathrm{SM})} g_{i}^{3}, \\
& \beta_{g_{i}}^{(\mathrm{KK})}=\frac{1}{(4 \pi)^{2}} b_{g_{i}}^{(\mathrm{KK})} g_{i}^{3},
\end{aligned}
$$

with $b_{g_{i}}^{(\mathrm{SM})}=\left(\frac{41}{6},-\frac{19}{6},-7\right)$ and $b_{g_{i}}^{(\mathrm{KK})}=\left(\frac{27}{2}, \frac{3}{2},-2\right)$ for $g_{i}=\left(g_{1}, g_{2}, g_{3}\right)$, respectively.

(iii) $\mathcal{Q}=y_{\ell_{k}}, y_{u_{k}}, y_{d_{k}}(k=1,2,3)$

$$
\begin{aligned}
\beta_{y_{\ell_{k}}}^{(\mathrm{SM})}= & \frac{1}{(4 \pi)^{2}}\left\{-\frac{15}{4} g_{1}^{2}-\frac{9}{4} g_{2}^{2}+\frac{3}{2} y_{\ell_{k}}^{2}\right. \\
& \left.+\sum_{i} N_{C_{f_{i}}} y_{f_{i}}^{2}\right\} y_{\ell_{k}}, \\
\beta_{y_{u_{k}}}^{\mathrm{SM})}= & \frac{1}{(4 \pi)^{2}}\left\{-\frac{17}{12} g_{1}^{2}-\frac{9}{4} g_{2}^{2}-8 g_{s}^{2}+\frac{3}{2} y_{u_{k}}^{2}\right. \\
& \left.+\sum_{j} y_{d_{j}}^{2}\left(V_{k j} V_{j k}^{\dagger}\right)+\sum_{i} N_{C_{f_{i}}} y_{f_{i}}^{2}\right\} y_{u_{k}}, \quad \\
\beta_{y_{d_{k}}}^{(\mathrm{SM})}= & \frac{1}{(4 \pi)^{2}}\left\{-\frac{15}{12} g_{1}^{2}-\frac{9}{4} g_{2}^{2}-8 g_{s}^{2}+\frac{3}{2} y_{d_{k}}^{2}\right. \\
& \left.+\sum_{j} y_{u_{j}}^{2}\left(V_{k_{j}}^{\dagger} V_{j k}\right)+\sum_{i} N_{C_{f_{i}}} y_{f_{i}}^{2}\right\} y_{d_{k}}, \quad(\mathrm{~A} 6) \\
\beta_{y_{\ell_{k}}}^{(\mathrm{KK})}= & \frac{1}{(4 \pi)^{2}}\left\{-\frac{9}{2} g_{1}^{2}-\frac{3}{2} g_{2}^{2}+\frac{3}{2} y_{\ell_{k}}^{2}\right. \\
\beta_{y_{d_{k}}}^{\mathrm{KK})}= & \frac{1}{(4 \pi)^{2}}\left\{-\frac{1}{18} g_{1}^{2}-\frac{3}{2} g_{2}^{2}-\frac{32}{3} g_{s}^{2}+\frac{3}{2} y_{d_{k}}^{2}\right. \\
& \left.+2 \sum_{i} N_{C_{f_{i}}} y_{C_{f_{i}}}^{2} y_{f_{i}}^{2}\right\} y_{\ell_{k}}, \\
\beta_{y_{u_{k}}}^{(\mathrm{KK})}= & \left.\frac{1}{(4 \pi)^{2}}\left\{-\frac{25}{18} g_{j}^{2}-\frac{3}{2} g_{2}^{2}-\frac{32}{3} g_{s}^{2}+\frac{3}{2} y_{u_{u_{k}}}^{\dagger} V_{j k}\right) y_{u_{j}}^{2}\right\} y_{d_{k}} . \\
& \left.+2 \sum_{i} N_{C_{f_{i}}} y_{f_{i}}^{2}-\frac{3}{2} \sum_{j}\left(V_{k j} V_{j k}^{\dagger}\right) y_{d_{j}}^{2}\right\} y_{u_{k^{\prime}}},
\end{aligned}
$$

\section{PS case}

In the case of PS, the contributions of the bosonic KK particles to the beta functions is classified into two categories as $\beta_{\mathrm{even}, \mathcal{Q}}^{(\mathrm{KK})}$ and $\beta_{\mathrm{odd}, Q}^{(\mathrm{KK})}$. (i) $\mathcal{Q}=\lambda$

$$
\begin{aligned}
\beta_{\mathrm{even}, \lambda}^{(\mathrm{KK})}= & \frac{1}{(4 \pi)^{2}}\left\{6 \lambda^{2}-3 \lambda g_{1}^{2}-9 \lambda g_{2}^{2}+2 g_{1}^{4}+4 g_{1}^{2} g_{2}^{2}\right. \\
& \left.+6 g_{2}^{4}+8 \sum_{i} \lambda N_{C_{f_{i}}} y_{f_{i}}^{2}-16 \sum_{i} N_{C_{f_{i}}} y_{f_{i}}^{4}\right\}
\end{aligned}
$$

$$
\begin{aligned}
\beta_{\mathrm{odd}, \lambda}^{(\mathrm{KK})}= & \frac{1}{(4 \pi)^{2}}\left\{+\frac{1}{2} g_{1}^{4}+g_{1}^{2} g_{2}^{2}+\frac{3}{2} g_{2}^{4}\right. \\
& \left.+8 \sum_{i} \lambda N_{C_{f_{i}}} y_{f_{i}}^{2}-16 \sum_{i} N_{C_{f_{i}}} y_{f_{i}}^{4}\right\}
\end{aligned}
$$

(ii) $\mathcal{Q}=g_{i}(i=1,2,3)$

$$
\begin{gathered}
\beta_{\mathrm{even}, g_{i}}^{(\mathrm{KK})}=\frac{1}{(4 \pi)^{2}} b_{\mathrm{even}, g_{i}}^{(\mathrm{KK})} g_{i}^{3}, \\
\beta_{\mathrm{odd}, g_{i}}^{(\mathrm{KK})}=\frac{1}{(4 \pi)^{2}} b_{\mathrm{odd}, g_{i}}^{(\mathrm{KK})} g_{i}^{3},
\end{gathered}
$$

with $b_{\text {even, } g_{i}}^{(\mathrm{KK})}=\left(\frac{27}{2}, \frac{7}{6},-\frac{5}{2}\right)$ and $b_{\text {odd }, g_{i}}^{(\mathrm{KK})}=\left(\frac{40}{3}, \frac{25}{3}, \frac{17}{2}\right)$ for $g_{i}=\left(g_{1}, g_{2}, g_{3}\right)$, respectively.

(iii) $\mathcal{Q}=y_{\ell_{k}}, y_{u_{k}}, y_{d_{k}}(k=1,2,3)$

$$
\begin{aligned}
\beta_{\mathrm{even}, y_{\ell_{k}}}^{(\mathrm{KK})}= & \frac{1}{(4 \pi)^{2}}\left\{-\frac{33}{8} g_{1}^{2}-\frac{15}{8} g_{2}^{2}+\frac{3}{2} y_{\ell_{k}}^{2}\right. \\
& \left.+2 \sum_{i} N_{C_{f_{i}}} y_{f_{i}}^{2}\right\} y_{\ell_{k}}, \\
\beta_{\mathrm{even}, y_{u_{k}}}^{(\mathrm{KK})}= & \frac{1}{(4 \pi)^{2}}\left\{-\frac{101}{72} g_{1}^{2}-\frac{15}{8} g_{2}^{2}-\frac{28}{3} g_{s}^{2}+\frac{3}{2} y_{u_{k}}^{2}\right. \\
& \left.+2 \sum_{i} N_{C_{f_{i}}} y_{f_{i}}^{2}-\frac{3}{2} \sum_{j}\left(V_{k j} V_{j k}^{\dagger}\right) y_{d_{j}}^{2}\right\} y_{u_{k}}, \\
\beta_{\mathrm{even}, y_{d_{k}}}^{(\mathrm{KK})}= & \frac{1}{(4 \pi)^{2}}\left\{-\frac{17}{72} g_{1}^{2}-\frac{15}{8} g_{2}^{2}-\frac{28}{3} g_{s}^{2}+\frac{3}{2} y_{d_{k}}^{2}\right. \\
& \left.+2 \sum_{i} N_{C_{f_{i}}} y_{f_{i}}^{2}-\frac{3}{2} \sum_{j}\left(V_{k j}^{\dagger} V_{j k}\right) y_{u_{j}}^{2}\right\} y_{d_{k}}, \\
\beta_{\mathrm{odd}, y_{\ell_{k}}}^{(\mathrm{KK})}= & \frac{1}{(4 \pi)^{2}}\left\{-\frac{3}{8} g_{1}^{2}+\frac{3}{8} g_{2}^{2}+2 \sum_{i} N_{C_{f_{i}}} y_{f_{i}}^{2}\right\} y_{\ell_{k}},
\end{aligned}
$$

$$
\begin{aligned}
\beta_{\mathrm{odd}, y_{u_{k}}}^{(\mathrm{KK})}= & \frac{1}{(4 \pi)^{2}}\left\{+\frac{1}{72} g_{1}^{2}+\frac{3}{8} g_{2}^{2}-\frac{4}{3} g_{s}^{2}\right. \\
& \left.+2 \sum_{i} N_{C_{f_{i}}} y_{f_{i}}^{2}\right\} y_{u_{k}},
\end{aligned}
$$




$$
\begin{aligned}
\beta_{\mathrm{odd}, y_{d_{k}}}^{(\mathrm{KK})}= & \frac{1}{(4 \pi)^{2}}\left\{+\frac{13}{72} g_{1}^{2}+\frac{3}{8} g_{2}^{2}-\frac{4}{3} g_{s}^{2}\right. \\
& \left.+2 \sum_{i} N_{C_{f_{i}}} y_{f_{i}}^{2}\right\} y_{d_{k}} .
\end{aligned}
$$

\section{3. $R P^{2}$ case}

In the regions having bosonic modes (regions I, II, and III), the following relations are fulfilled for each type of coupling $C$ :

$\beta_{\text {regionI }, C}^{(\mathrm{KK})}=\beta_{\text {even }, C}^{(\mathrm{KK})}, \quad \beta_{\text {regionII, } C}^{(\mathrm{KK})}=\beta_{\mathrm{odd}, C}^{(\mathrm{KK})}, \quad \beta_{\text {region III, } C}^{(\mathrm{KK})}=\beta_{C}^{(\mathrm{KK})}$.

We write down the formula for the region without having a bosonic mode (region IV).

(i) $\mathcal{Q}=\lambda$

$$
\beta_{\text {region IV, } \lambda}^{(\mathrm{KK})}=\frac{1}{(4 \pi)^{2}}\left\{+8 \sum_{i} \lambda N_{C_{f_{i}}} y_{f_{i}}^{2}-16 \sum_{i} N_{C_{f_{i}}} y_{f_{i}}^{4}\right\} .
$$

(ii) $\mathcal{Q}=g_{i}(i=1,2,3)$

$$
\beta_{\text {region IV, } g_{i}}^{(\mathrm{KK})}=\frac{1}{(4 \pi)^{2}} b_{\text {region IV, } g_{i}}^{(\mathrm{KK})} g_{i}^{3},
$$

with $b_{\text {region IV, } g_{i}}^{(\mathrm{KK})}=\left(\frac{40}{3}, 8,8\right)$ for $g_{i}=\left(g_{1}, g_{2}, g_{3}\right)$, respectively.

(iii) $\mathcal{Q}=y_{\ell_{k}}, y_{u_{k}}, y_{d_{k}}(k=1,2,3)$

$$
\begin{aligned}
& \beta_{\text {region IV }, y_{\ell_{k}}}^{(\mathrm{KK})}=\frac{1}{(4 \pi)^{2}}\left\{+2 \sum_{i} N_{C_{f_{i}}} y_{f_{i}}^{2}\right\} y_{\ell_{k}}, \\
& \beta_{\text {region IV }, y_{u_{k}}}^{(\mathrm{KK})}=\frac{1}{(4 \pi)^{2}}\left\{+2 \sum_{i} N_{C_{f_{i}}} y_{f_{i}}^{2}\right\} y_{u_{k}}, \\
& \beta_{\text {region IV }, y_{d_{k}}}^{\text {(KK) }}=\frac{1}{(4 \pi)^{2}}\left\{+2 \sum_{i} N_{C_{f_{i}}} y_{f_{i}}^{2}\right\} y_{d_{k}} .
\end{aligned}
$$

\section{4. mUED case}

The surviving modes for each KK level in the mUED are totally the same as in region I of the $R P^{2}$ or in the "even" region of the PS. Hence, we can use those forms for RGEs in the mUED. We can check that our results of this part are consistent with those in Refs. [97,98].

\section{APPENDIX B: LOOP FUNCTIONS IN SINGLE HIGGS PRODUCTION AND DECAY}

In this appendix, we summarize the loop functions that are needed for estimating the single Higgs production through the gluon fusion process and the Higgs decay into a pair of photons. Readers who want more explanations on the above expressions should consult Ref. [55].
For each model, the loop function $J_{t}^{\text {model }}$ describes the contributions of all the zero and KK modes for the top quark in the triangle loops:

$$
J_{t}^{\mathrm{SM}}(\hat{s})=I\left(\frac{m_{t}^{2}}{\hat{s}}\right),
$$

$$
J_{t}^{\operatorname{mUED}}(\hat{s})=\left\{I\left(\frac{m_{t}^{2}}{\hat{s}}\right)+2 \sum_{n \geq 1}\left(\frac{m_{t}}{m_{t(n)}}\right)^{2} I\left(\frac{m_{t(n)}^{2}}{\hat{s}}\right)\right\}
$$

$$
\begin{aligned}
J_{t}^{T^{2} / Z_{2}}(\hat{s}) & =J_{t}^{R P^{2}}(\hat{s}) \\
& =\left\{I\left(\frac{m_{t}^{2}}{\hat{s}}\right)+2 \sum_{\substack{m+n \geq 1 \\
o r m=-n \geq 1}}\left(\frac{m_{t}}{m_{t(m, n)}}\right)^{2} I\left(\frac{m_{t(m, n)}^{2}}{\hat{s}}\right)\right\},
\end{aligned}
$$

$$
\begin{aligned}
& J_{t}^{T^{2} / Z_{4}}(\hat{s})=\left\{I\left(\frac{m_{t}^{2}}{\hat{s}}\right)+2 \sum_{m \geq 1, n \geq 0}\left(\frac{m_{t}}{m_{t(m, n)}}\right)^{2} I\left(\frac{m_{t(m, n)}^{2}}{\hat{s}}\right)\right\}, \quad(\mathrm{B} 4) \\
& J_{t}^{T^{2} /\left(Z_{2} \times Z_{2}^{\prime}\right)}(\hat{s})=\left\{I\left(\frac{m_{t}^{2}}{\hat{s}}\right)+2 \sum_{\substack{m \geq 0, n \geq 0 \\
(m, n) \neq(0,0)}}\left(\frac{m_{t}}{m_{t(m, n)}}\right)^{2} I\left(\frac{m_{t(m, n)}^{2}}{\hat{s}}\right)\right\},
\end{aligned}
$$

$$
J_{t}^{S^{2} / Z_{2}}(\hat{s})=\left\{I\left(\frac{m_{t}^{2}}{\hat{s}}\right)+2 \sum_{j \geq 1}\left(\frac{m_{t}}{m_{t(j)}}\right)^{2} n^{S^{2} / Z_{2}}(j) I\left(\frac{m_{t(j)}^{2}}{\hat{s}}\right)\right\}
$$

$$
\begin{aligned}
J_{t}^{\mathrm{PS}}(\hat{s}) & =J_{t}^{S^{2}}(\hat{s}) \\
& =\left\{I\left(\frac{m_{t}^{2}}{\hat{s}}\right)+2 \sum_{j \geq 1}\left(\frac{m_{t}}{m_{t(j)}}\right)^{2}(2 j+1) I\left(\frac{m_{t(j)}^{2}}{\hat{s}}\right)\right\},
\end{aligned}
$$

where $I$ is given by

$$
I(\lambda)=-2 \lambda+\lambda(1-4 \lambda) \int_{0}^{1} \frac{d x}{x} \ln \left[\frac{x(x-1)}{\lambda}+1-i \epsilon\right] .
$$

The explicit result of the integral is

$$
\begin{aligned}
\int_{0}^{1} \frac{d x}{x} \ln \left[\frac{x(x-1)}{\lambda}+1-i \epsilon\right] \\
= \begin{cases}-2\left[\arcsin \frac{1}{\sqrt{4 \lambda}}\right]^{2} & \left(\text { for } \lambda \geq \frac{1}{4}\right), \\
\frac{1}{2}\left[\ln \frac{1+\sqrt{1-4 \lambda}}{1-\sqrt{1-4 \lambda}}-i \pi\right]^{2} & \left(\text { for } \lambda<\frac{1}{4}\right),\end{cases}
\end{aligned}
$$

where this form is related with the Passarino-Veltman three-point scalar function $C_{0}[120] . n^{\text {model }}(j)$ counts the number of degeneracy, and the explicit forms are shown in Eqs. (10)-(12), and we write the KK top and $W$ masses $(X=t, W)$ :

$$
m_{X(n)} \equiv \sqrt{m_{X}^{2}+\frac{n^{2}}{R^{2}}}
$$




$$
\begin{gathered}
m_{X(m, n)} \equiv \sqrt{m_{X}^{2}+\frac{m^{2}}{R_{5}^{2}}+\frac{n^{2}}{R_{6}^{2}}} \\
m_{X(j)} \equiv \sqrt{m_{X}^{2}+\frac{j(j+1)}{R^{2}}}
\end{gathered}
$$

The range of the KK summation reflects the structure of each extra-dimensional background. ${ }^{8}$ The loop functions that are needed for the process $H \rightarrow \gamma \gamma$ are as follows:

$$
\begin{gathered}
J_{W}^{\mathrm{SM}}\left(m_{H}^{2}\right)=L\left(\frac{1}{2}, 3,3,6,0 ; \frac{m_{W}^{2}}{m_{H}^{2}}, \frac{m_{W}^{2}}{m_{H}^{2}}\right), \\
J_{W}^{\mathrm{mUED}}\left(m_{H}^{2}\right)=J_{W}^{\mathrm{SM}}\left(m_{H}^{2}\right)+\sum_{n \geq 1} L\left(\frac{1}{2}, 4,4,8,1 ; \frac{m_{W}^{2}}{m_{H}^{2}}, \frac{m_{W(n)}^{2}}{m_{H}^{2}}\right),
\end{gathered}
$$

$$
\begin{aligned}
J_{W}^{T^{2} / Z_{4}}\left(m_{H}^{2}\right)= & J_{W}^{\mathrm{SM}}\left(m_{H}^{2}\right) \\
& +\sum_{m \geq 1, n \geq 0} L\left(\frac{1}{2}, 5,4,10,1 ; \frac{m_{W}^{2}}{m_{H}^{2}}, \frac{m_{W(m, n)}^{2}}{m_{H}^{2}}\right),
\end{aligned}
$$

$$
\begin{aligned}
J_{W}^{T^{2} /\left(Z_{2} \times Z_{2}^{\prime}\right)}\left(m_{H}^{2}\right)= & J_{W}^{\mathrm{SM}}\left(m_{H}^{2}\right) \\
& +\sum_{\substack{m \geq 0, n \geq 0 \\
(m, n) \neq(0,0)}} L\left(\frac{1}{2}, 5,4,10,1 ; \frac{m_{W}^{2}}{m_{H}^{2}}, \frac{m_{W(m, n)}^{2}}{m_{H}^{2}}\right),
\end{aligned}
$$

$$
\begin{aligned}
J_{W}^{T^{2} / Z_{2}}\left(m_{H}^{2}\right)= & J_{W}^{\mathrm{SM}}\left(m_{H}^{2}\right) \\
& +\sum_{\substack{m+n \geq 1 \\
\text { or } m=-n \geq 1}} L\left(\frac{1}{2}, 5,4,10,1 ; \frac{m_{W}^{2}}{m_{H}^{2}}, \frac{m_{W(m, n)}^{2}}{m_{H}^{2}}\right),
\end{aligned}
$$

$$
\begin{aligned}
J_{W}^{R P^{2}}\left(m_{H}^{2}\right)= & J_{W}^{\mathrm{SM}}\left(m_{H}^{2}\right)+\sum_{(m, n)}^{A} L\left(\frac{1}{2}, 4,4,8,1 ; \frac{m_{W}^{2}}{m_{H}^{2}}, \frac{m_{W(m, n)}^{2}}{m_{H}^{2}}\right) \\
& +\sum_{(m, n)}^{B} L\left(0,1,0,2,0 ; \frac{m_{W}^{2}}{m_{H}^{2}}, \frac{m_{W(m, n)}^{2}}{m_{H}^{2}}\right),
\end{aligned}
$$

$$
\begin{aligned}
J_{W}^{S^{2} / Z_{2}}\left(m_{H}^{2}\right)= & J_{W}^{\mathrm{SM}}\left(m_{H}^{2}\right) \\
& +\sum_{j \geq 1} n^{S^{2} / Z_{2}}(j) L\left(\frac{1}{2}, 5,4,10,1 ; \frac{m_{W}^{2}}{m_{H}^{2}}, \frac{m_{W(j)}^{2}}{m_{H}^{2}}\right),
\end{aligned}
$$

\footnotetext{
${ }^{8}$ The origin of the factor 2 in front of each KK summation is the fact that there are both left- and right-handed (namely, vectorlike) KK modes for each chiral quark zero mode corresponding to a SM quark.
}

$$
\begin{aligned}
J_{W}^{S^{2}}\left(m_{H}^{2}\right)= & J_{W}^{\mathrm{SM}}\left(m_{H}^{2}\right) \\
& +\sum_{j \geq 1}(2 j+1) L\left(\frac{1}{2}, 5,4,10,1 ; \frac{m_{W}^{2}}{m_{H}^{2}}, \frac{m_{W(j)}^{2}}{m_{H}^{2}}\right),
\end{aligned}
$$

$$
\begin{aligned}
J_{W}^{\mathrm{PS}}\left(m_{H}^{2}\right)= & J_{W}^{\mathrm{SM}}\left(m_{H}^{2}\right)+\sum_{j \geq 1}\left[n_{\mathrm{even}}^{\mathrm{PS}}(j) L\left(\frac{1}{2}, 4,4,8,1 ; \frac{m_{W}^{2}}{m_{H}^{2}}, \frac{m_{W(j)}^{2}}{m_{H}^{2}}\right)\right. \\
& \left.+n_{\text {odd }}^{\mathrm{PS}}(j) L\left(0,1,0,2,0 ; \frac{m_{W}^{2}}{m_{H}^{2}}, \frac{m_{W(j)}^{2}}{m_{H}^{2}}\right)\right], \quad(\mathrm{B} 21)
\end{aligned}
$$

with

$$
\begin{aligned}
L\left(a, b, c, d, e ; \lambda_{1}, \lambda_{2}\right)= & a+b \lambda_{1}-\left[\lambda_{1}\left(c-d \lambda_{2}\right)-e \lambda_{2}\right] \\
& \times \int_{0}^{1} \frac{d x}{x} \ln \left[\frac{x(x-1)}{\lambda_{2}}+1-i \epsilon\right] .
\end{aligned}
$$

The $A$ summation for $R P^{2}$ are over the region that satisfies both $m \geq 1$ and $n \geq 1$ as well as over the ranges $(m, n)=$ $(0,2),(0,4),(0,6), \ldots$ and $(m, n)=(2,0),(4,0),(6,0), \ldots$. Similarly, the $B$ summation is over $m \geq 1$ and $n \geq 1$ as well as over $(m, n)=(0,1),(0,3),(0,5), \ldots$ and $(m, n)=$ $(1,0),(3,0),(5,0), \ldots$.

\section{APPENDIX C: TWO-POINT FUNCTIONS OF GAUGE BOSONS IN 6D UEDS}

In this section, we summarize the two-point functions of photons, $\mathrm{W}$, and $\mathrm{Z}$ bosons for calculating Peskin-Takeuchi $S$ and $T$ parameters.

\section{Notations}

First, we summarize our notations for the PassarinoVeltman B function [120]. In this section, we use the following descriptions for masses. The mass squared of the "sth" KK mode of the particle $X$ is represented as

$$
M_{X_{s}}^{2}=m_{X}^{2}+M_{s}^{2},
$$

where $m_{X}$ is the corresponding zero-mode mass, and $M_{s}$ is the $s$ th level KK mass. Since only $Z, W, H$, and top masses are not negligible compared with the KK scale $M_{s}$, we use the representations

$$
\begin{aligned}
M_{W_{s}}^{2}:=m_{W}^{2}+M_{s}^{2}, & M_{Z_{s}}^{2}:=m_{Z}^{2}+M_{s}^{2} \\
M_{t_{s}}^{2}:=m_{t}^{2}+M_{s}^{2}, & M_{H_{s}}^{2}:=m_{H}^{2}+M_{s}^{2},
\end{aligned}
$$

and for the other fields,

$$
M_{X_{s}}^{2} \simeq M_{s}^{2}
$$

We will use the Passarino-Veltman loop integral to calculate two-point functions of the gauge bosons with external momentum $k$ below, and the definition is 


$$
\begin{aligned}
\frac{1}{(4 \pi)^{2}} B_{X_{s}, Y_{s}}\left(k^{2}\right)= & \int \frac{d^{d} p}{(2 \pi)^{d}} \frac{1}{\left(p^{2}-M_{X_{s}}^{2}\right)\left((p+k)^{2}-M_{Y_{s}}^{2}\right)} \\
= & \frac{i}{(4 \pi)^{2}}\left\{\frac{1}{\bar{\varepsilon}}-\int_{0}^{1} d x \ln \left[(1-x) M_{X_{s}}^{2}\right.\right. \\
& \left.\left.+x M_{Y_{s}}^{2}-x(1-x) k^{2}-i \epsilon\right]\right\},
\end{aligned}
$$

where we use the dimensional regularization in $d$ dimensions, and $\epsilon$ is an infinitesimal positive value. $1 / \bar{\varepsilon}(:=1 / \varepsilon-\gamma+\ln 4 \pi)$ means the usual common divergent part with $\varepsilon=2-d / 2$ and the Euler-Mascheroni constant $\gamma$. The following short-hand description is also used later for simplicity:

$$
\begin{aligned}
B_{X_{s}}\left(k^{2}\right) & :=B_{X_{s}, X_{s}}\left(k^{2}\right), \\
\delta B_{X_{s}, Y_{s}}\left(k^{2}\right) & :=\frac{B_{X_{s}, Y_{s}}\left(k^{2}\right)-B_{X_{s}, Y_{s}}(0)}{k^{2}} .
\end{aligned}
$$

Here, we write down some useful formulas for calculations:

$$
\begin{gathered}
B_{X_{s}, Y_{s}}(0) \simeq \frac{1}{\bar{\varepsilon}}-\frac{1}{2} \frac{m_{X}^{2}+m_{Y}^{2}}{M_{s}^{2}}, \\
B_{X_{s}, Y_{s}}^{\prime}(0) \simeq \frac{1}{6 M_{s}^{2}}, \\
B_{X_{s}, Y_{s}^{\prime \prime}}^{\prime \prime}(0) \simeq \frac{2}{3} \frac{1}{\left(m_{X}^{2}-m_{Y}^{2}\right)^{2}} \frac{m_{X}^{2}+m_{Y}^{2}}{M_{s}^{2}},
\end{gathered}
$$

where we assume the hierarchy $m_{X}^{2}, m_{Y}^{2} \ll M_{s}^{2}$, and values with a prime mean that it is differentiated with respect to $k^{2}$ once.

\section{Bosonic contributions to the two-point function of gauge bosons in 6D UEDs abd mUED}

In this section, we make a summary of bosonic twopoint contributions to the two-point function of gauge bosons in the 6D UEDs and the mUED for evaluating $S$ and $T$ parameters. For contributions of fermions, we can use the result in Ref. [88].

The generic form of a gauge boson two-point function is as follows:

$\Pi_{a b}^{\mu \nu}\left(k^{2}\right)=i \Pi_{a b}^{\mathrm{T}}\left(k^{2}\right)\left(g^{\mu \nu}-\frac{k^{\mu} k^{\nu}}{k^{2}}\right)+i \Pi_{a b}^{\mathrm{L}}\left(k^{2}\right) \frac{k^{\mu} k^{\nu}}{k^{2}}$,

where $a$ and $b$ show the type of gauge bosons, and the superscript T (L) indicates the transverse (longitudinal), respectively.

For estimating the $S$ and $T$ parameters, we calculate only the transverse ones. In each following subsection, we show the contributions of KK bosonic particles to the two-point functions from the level-s KK states.

\section{a. UEDs on oriented geometry case}

$$
\begin{aligned}
\Pi_{\gamma \gamma}^{\mathrm{T}, s}\left(k^{2}\right)= & \frac{\alpha}{4 \pi}\left\{-\frac{4}{9} k^{2}+\left(\frac{7}{3} k^{2}+\frac{20}{3} M_{W_{s}}^{2}\right) B_{W_{s}}\left(k^{2}\right)\right. \\
& \left.-\frac{20}{3} M_{W_{s}}^{2} B_{W_{s}}(0)\right\}, \quad(\mathrm{C} 10) \\
\Pi_{Z \gamma}^{\mathrm{T}, s}\left(k^{2}\right)= & \frac{\alpha}{4 \pi s_{W} c_{W}}\left\{\left(-\frac{1}{9}+\frac{4}{9} c_{W}^{2}\right) k^{2}\right. \\
& +\left(\frac{20}{3} c_{W}^{2}-\frac{2}{3}\right) M_{W_{s}}^{2} B_{W_{s}}(0)+\left[\left(-\frac{1}{6}-\frac{7}{3} c_{W}^{2}\right) k^{2}\right. \\
& \left.\left.-\left(\frac{20}{3} c_{W}^{2}-\frac{2}{3}\right) M_{W_{s}}^{2}-2 m_{W}^{2}\right] B_{W_{s}}\left(k^{2}\right)\right\}, \quad(\mathrm{C} 11)
\end{aligned}
$$

$$
\begin{aligned}
\Pi_{Z Z}^{\mathrm{T}, s}\left(k^{2}\right)= & \frac{\alpha}{4 \pi s_{W}^{2} c_{W}^{2}}\left\{\left(\frac{2}{9} c_{W}^{2}-\frac{4}{9} c_{W}^{4}-\frac{1}{18}\right) k^{2}+\left(-\frac{20}{3} c_{W}^{4}+\frac{4}{3} c_{W}^{2}-\frac{1}{3}\right) M_{W_{s}}^{2} B_{W_{s}}(0)-\frac{1}{6} M_{H_{s}}^{2} B_{H_{s}}(0)-\frac{1}{6} M_{Z_{s}}^{2} B_{Z_{s}}(0)\right. \\
& +\left[\left(\frac{7}{3} c_{W}^{4}+\frac{1}{3} c_{W}^{2}-\frac{1}{12}\right) k^{2}+\left(\frac{20}{3} c_{W}^{4}-\frac{4}{3} c_{W}^{2}+\frac{1}{3}\right) M_{W_{s}}^{2}-\left(2-4 c_{W}^{2}\right) m_{W}^{2}\right] B_{W_{s}}\left(k^{2}\right) \\
& \left.+\left(-\frac{1}{12} k^{2}+\frac{1}{6} M_{Z_{s}}^{2}+\frac{1}{6} M_{H_{s}}^{2}-\frac{m_{Z}^{2}}{c_{W}^{2}}\right) B_{H_{s}, Z_{s}}\left(k^{2}\right)-\frac{1}{12}\left(M_{H_{s}}^{2}-M_{Z_{s}}^{2}\right)^{2} \delta B_{H_{s}, Z_{s}}\left(k^{2}\right)\right\}, \\
\Pi_{W W}^{\mathrm{T}, s}\left(k^{2}\right)= & \frac{\alpha}{4 \pi s_{W}^{2}}\left\{-\frac{1}{3} k^{2}-\frac{1}{6} M_{H_{s}}^{2} B_{H_{s}}(0)-3 M_{W_{s}}^{2} B_{W_{s}}(0)-\frac{17}{6} M_{Z_{s}}^{2} B_{Z_{s}}(0)+\left[-\frac{1}{12} k^{2}+\frac{1}{6} M_{H_{s}}^{2}+\frac{1}{6} M_{W_{s}}^{2}-m_{W}^{2}\right] B_{H_{s}, W_{s}}\left(k^{2}\right)\right. \\
& +\left[\frac{31}{12} k^{2}+\frac{23}{6}\left(M_{W_{s}}^{2}+M_{Z_{s}}^{2}\right)-2 M_{s}^{2}-m_{Z}^{2}\left(c_{W}^{2}-2+c_{W}^{-2}\right)\right] B_{W_{s}, Z_{s}}\left(k^{2}\right) \\
& \left.-\frac{1}{12}\left(M_{H_{s}}^{2}-M_{W_{s}}^{2}\right)^{2} \delta B_{H_{s}, W_{s}}\left(k^{2}\right)-\frac{17}{12}\left(M_{Z_{s}}^{2}-M_{W_{s}}^{2}\right)^{2} \delta B_{Z_{s}, W_{s}}\left(k^{2}\right)\right\} .
\end{aligned}
$$

\section{b. $R P^{2}, P S$, and $m U E D$ cases}

As we have discussed in Sec. II, the particle contents of region III of the $R P^{2}$ model is completely the same as those of the 6D UEDs on oriented geometries just as above, and we need not discuss them. Based on the knowledge in Sec. II, the remaining boson contributions are written down as follows: 

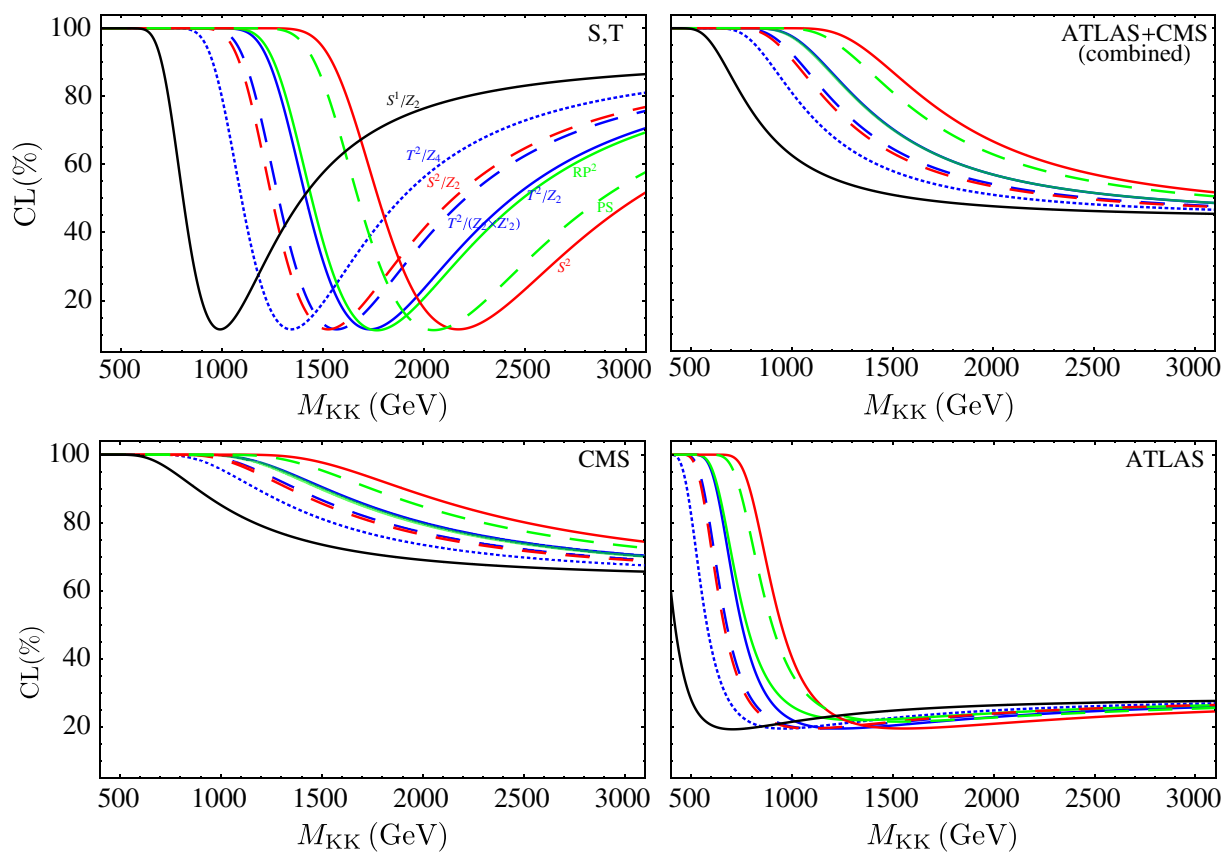

FIG. 10 (color online). The exclusion C.L.s of all the UED models as functions of the KK scale $M_{\mathrm{KK}}$ obtained from the experimental results of the Higgs searches at the LHC (ATLAS, CMS, and both of them, respectively) and those of $S, T$ parameters. Colors denote the same as in Fig. 2.

$$
\begin{gathered}
\left.\Pi_{\gamma \gamma}^{\mathrm{T}, s}\right|_{\text {region II }}\left(k^{2}\right)=\left.\Pi_{\gamma \gamma}^{\mathrm{T}, s}\right|_{\text {odd }}\left(k^{2}\right)=\frac{\alpha}{4 \pi}\left\{-\frac{2}{9} k^{2}+\left(-\frac{1}{3} k^{2}+\frac{4}{3} M_{W_{s}}^{2}\right) B_{W_{s}}\left(k^{2}\right)-\frac{4}{3} M_{W_{s}}^{2} B_{W_{s}}(0)\right\}, \\
\left.\Pi_{Z \gamma}^{\mathrm{T}, s}\right|_{\text {region II }}\left(k^{2}\right)=\left.\Pi_{Z \gamma}^{\mathrm{T}, s}\right|_{\text {odd }}\left(k^{2}\right)=\frac{\alpha}{4 \pi}\left[-\frac{c_{W}}{s_{W}}\right]\left\{-\frac{2}{9} k^{2}+\left(-\frac{1}{3} k^{2}+\frac{4}{3} M_{W_{s}}^{2}\right) B_{W_{s}}\left(k^{2}\right)-\frac{4}{3} M_{W_{s}}^{2} B_{W_{s}}(0)\right\}, \\
\left.\Pi_{Z Z}^{\mathrm{T}, s}\right|_{\text {region II }}\left(k^{2}\right)=\left.\Pi_{Z Z}^{\mathrm{T}, s}\right|_{\text {odd }}\left(k^{2}\right)=\frac{\alpha}{4 \pi}\left[\frac{c_{W}^{2}}{s_{W}^{2}}\right]\left\{-\frac{2}{9} k^{2}+\left(-\frac{1}{3} k^{2}+\frac{4}{3} M_{W_{s}}^{2}\right) B_{W_{s}}\left(k^{2}\right)-\frac{4}{3} M_{W_{s}}^{2} B_{W_{s}}(0)\right\}, \\
\left.\Pi_{W W}^{\mathrm{T}, s}\right|_{\text {region II }}\left(k^{2}\right)=\left.\Pi_{W W}^{\mathrm{T}, s}\right|_{\text {odd }}\left(k^{2}\right) \\
=\frac{\alpha}{4 \pi s_{W}^{2}}\left\{-\frac{2}{9} k^{2}-\frac{2}{3} M_{W_{s}}^{2} B_{W_{s}}(0)-\frac{2}{3} M_{Z_{s}}^{2} B_{Z_{s}}(0)+\left[-\frac{1}{3} k^{2}+\frac{2}{3}\left(M_{W_{s}}^{2}+M_{Z_{s}}^{2}\right)\right] B_{W_{s}, Z_{s}}\left(k^{2}\right)\right. \\
\left.-\frac{1}{3}\left(M_{Z_{s}}^{2}-M_{W_{s}}^{2}\right)^{2} \delta B_{Z_{s}, W_{s}}\left(k^{2}\right)\right\} .
\end{gathered}
$$

The remaining part can be easily calculated by use of the following relations:

$$
\begin{gathered}
\left.\Pi_{a b}^{\mathrm{T}, s}\right|_{\text {region I }}\left(k^{2}\right)=\Pi_{a b}^{\mathrm{T}, s}\left(k^{2}\right)-\left.\Pi_{a b}^{\mathrm{T}, s}\right|_{\text {region II }}\left(k^{2}\right), \\
\left.\Pi_{a b}^{\mathrm{T}, s}\right|_{\text {even }}\left(k^{2}\right)=\Pi_{a b}^{\mathrm{T}, s}\left(k^{2}\right)-\left.\Pi_{a b}^{\mathrm{T}, s}\right|_{\text {odd }}\left(k^{2}\right),
\end{gathered}
$$

where $a b$ represents the possible four combinations of gauge bosons.
We also derive the following relations for the mUED:

$$
\left.\left.\Pi_{a b}^{\mathrm{T}, s}\right|_{\mathrm{mUED}}\left(k^{2}\right) \simeq \Pi_{a b}^{\mathrm{T}, s}\right|_{\mathrm{even}}\left(k^{2}\right),
$$

based on the discussions in Secs. II and IV.

\section{APPENDIX D: SUMMARY OF THE BOUNDS}

Here, we summarize the bounds on the KK scale in UED models. Figure 10 shows the exclusion C.L.s as functions of the wide range of the $\mathrm{KK}$ scale. 
[1] G. Aad et al. (ATLAS Collaboration), Phys. Lett. B 716, 1 (2012).

[2] S. Chatrchyan et al. (CMS Collaboration), Phys. Lett. B 716, 30 (2012).

[3] The ATLAS Collaboration, Report No. ATLAS-CONF2013-012, 2013.

[4] The ATLAS Collaboration, Report No. ATLAS-CONF2013-013, 2013.

[5] The ATLAS Collaboration, Report No. ATLAS-CONF2013-030, 2013.

[6] The CMS Collaboration, Report No. CMS PAS HIG-13001, 2013.

[7] The CMS Collaboration, Report No. CMS PAS HIG-13002, 2013.

[8] The CMS Collaboration, Report No. CMS PAS HIG-13003, 2013.

[9] A. Azatov, R. Contino, and J. Galloway, J. High Energy Phys. 04 (2012) 127.

[10] A. David et al. (L.H.C.S.W. Group), arXiv:1209.0040.

[11] G. Moreau, Phys. Rev. D 87, 015027 (2013).

[12] G. Cacciapaglia, A. Deandrea, G. D. La Rochelle, and J.-B. Flament, J. High Energy Phys. 03 (2013) 029.

[13] A. Falkowski, F. Riva, and A. Urbano, arXiv:1303.1812.

[14] P. P. Giardino, K. Kannike, I. Masina, M. Raidal, and A. Strumia, arXiv:1303.3570.

[15] J. Ellis and T. You, arXiv:1303.3879.

[16] A. Djouadi and G. Moreau, arXiv:1303.6591.

[17] G. Bertone, K. Kong, R. R. de Austri, and R. Trotta, Phys. Rev. D 83, 036008 (2011).

[18] T. Appelquist, H.-C. Cheng, and B. A. Dobrescu, Phys. Rev. D 64, 035002 (2001).

[19] I. Antoniadis, Phys. Lett. B 246, 377 (1990).

[20] G. Servant and T. M. Tait, Nucl. Phys. B650, 391 (2003).

[21] G. Belanger, M. Kakizaki, and A. Pukhov, J. Cosmol. Astropart. Phys. 02 (2011) 009.

[22] M. Baak, M. Goebel, J. Haller, A. Hoecker, D. Kennedy, K. Mönig, M. Schott, and J. Stelzer, Eur. Phys. J. C 72, 2003 (2012).

[23] U. Haisch and A. Weiler, Phys. Rev. D 76, 034014 (2007).

[24] T. G. Rizzo, Phys. Rev. D 64, 095010 (2001).

[25] C. Macesanu, C. McMullen, and S. Nandi, Phys. Rev. D 66, 015009 (2002)

[26] H.-C. Cheng, K. T. Matchev, and M. Schmaltz, Phys. Rev. D 66, 056006 (2002).

[27] C. D. Carone, J. M. Conroy, M. Sher, and I. Turan, Phys. Rev. D 69, 074018 (2004).

[28] G. Bhattacharyya, P. Dey, A. Kundu, and A. Raychaudhuri, Phys. Lett. B 628, 141 (2005).

[29] B. Bhattacherjee and A. Kundu, Phys. Lett. B 627, 137 (2005).

[30] J. A. Cembranos, J. L. Feng, and L. E. Strigari, Phys. Rev. D 75, 036004 (2007).

[31] B. Bhattacherjee and A. Kundu, Phys. Lett. B 653, 300 (2007).

[32] B. Bhattacherjee, A. Kundu, S.K. Rai, and S. Raychaudhuri, Phys. Rev. D 78, 115005 (2008).

[33] P. Konar, K. Kong, K. T. Matchev, and M. Perelstein, New J. Phys. 11, 105004 (2009).

[34] S. Matsumoto, J. Sato, M. Senami, and M. Yamanaka, Phys. Rev. D 80, 056006 (2009).
[35] G. Bhattacharyya, A. Datta, S. K. Majee, and A. Raychaudhuri, Nucl. Phys. B821, 48 (2009).

[36] P. Bandyopadhyay, B. Bhattacherjee, and A. Datta, J. High Energy Phys. 03 (2010) 048.

[37] D. Choudhury, A. Datta, and K. Ghosh, J. High Energy Phys. 08 (2010) 051.

[38] B. Bhattacherjee and K. Ghosh, Phys. Rev. D 83, 034003 (2011).

[39] H. Murayama, M. M. Nojiri, and K. Tobioka, Phys. Rev. D 84, 094015 (2011).

[40] A. Datta, A. Datta, and S. Poddar, Phys. Lett. B 712, 219 (2012).

[41] S. Chang, K. Y. Lee, S. Y. Shim, and J. Song, Phys. Rev. D 86, 117503 (2012).

[42] A. Belyaev, M. Brown, J. Moreno, and C. Papineau, J. High Energy Phys. 06 (2013) 080.

[43] L. Edelhauser, T. Flacke, and M. Kramer, arXiv:1302.6076.

[44] M. Battaglia, A. Datta, A. De Roeck, K. Kong, and K. T. Matchev, J. High Energy Phys. 07 (2005) 033.

[45] J. M. Smillie and B. R. Webber, J. High Energy Phys. 10 (2005) 069.

[46] A. Datta, K. Kong, and K. T. Matchev, Phys. Rev. D 72, 096006 (2005).

[47] A. Datta, G. L. Kane, and M. Toharia, arXiv:hep-ph/ 0510204.

[48] B. Bhattacherjee, A. Kundu, S. K. Rai, and S. Raychaudhuri, Phys. Rev. D 81, 035021 (2010).

[49] K. Ghosh, S. Mukhopadhyay, and B. Mukhopadhyaya, J. High Energy Phys. 10 (2010) 096.

[50] B. A. Dobrescu and E. Ponton, J. High Energy Phys. 03 (2004) 071

[51] G. Burdman, B. A. Dobrescu, and E. Ponton, J. High Energy Phys. 02 (2006) 033.

[52] R. N. Mohapatra and A. Perez-Lorenzana, Phys. Rev. D 67, 075015 (2003).

[53] N. Maru, T. Nomura, J. Sato, and M. Yamanaka, Nucl. Phys. B830, 414 (2010).

[54] K. Nishiwaki, K.-y. Oda, N. Okuda, and R. Watanabe, Phys. Lett. B 707, 506 (2012).

[55] K. Nishiwaki, K.-y. Oda, N. Okuda, and R. Watanabe, Phys. Rev. D 85, 035026 (2012).

[56] G. Cacciapaglia, A. Deandrea, and J. Llodra-Perez, J. High Energy Phys. 03 (2010) 083.

[57] H. Dohi and K.-y. Oda, Phys. Lett. B 692, 114 (2010).

[58] B. A. Dobrescu and E. Poppitz, Phys. Rev. Lett. 87, 031801 (2001).

[59] G. Burdman, B. A. Dobrescu, and E. Ponton, Phys. Rev. D 74, 075008 (2006).

[60] B. A. Dobrescu, K. Kong, and R. Mahbubani, J. High Energy Phys. 07 (2007) 006.

[61] K. Ghosh and A. Datta, Nucl. Phys. B800, 109 (2008).

[62] K. Ghosh and A. Datta, Phys. Lett. B 665, 369 (2008).

[63] K. Ghosh, J. High Energy Phys. 04 (2009) 049.

[64] D. Choudhury, A. Datta, D. K. Ghosh, and K. Ghosh, J. High Energy Phys. 04 (2012) 057.

[65] G. Cacciapaglia, A. Deandrea, and J. Llodra-Perez, J. High Energy Phys. 10 (2011) 146.

[66] G. Cacciapaglia, R. Chierici, A. Deandrea, L. Panizzi, S. Perries, and S. Tosi, J. High Energy Phys. 10 (2011) 042. 
[67] G. Cacciapaglia and B. Kubik, J. High Energy Phys. 02 (2013) 052.

[68] G. Cacciapaglia, A. Deandrea, J. Ellis, J. Marrouche, and L. Panizzi, Phys. Rev. D 87, 075006 (2013).

[69] B. Bhattacherjee, Phys. Rev. D 79, 016006 (2009).

[70] N. Haba, K.-y. Oda, and R. Takahashi, Nucl. Phys. B821, 74 (2009).

[71] S. C. Park and J. Shu, Phys. Rev. D 79, 091702 (2009).

[72] C.-R. Chen, M.M. Nojiri, S.C. Park, J. Shu, and M. Takeuchi, J. High Energy Phys. 09 (2009) 078.

[73] K. Kong, S. C. Park, and T. G. Rizzo, J. High Energy Phys. 04 (2010) 081.

[74] G.-Y. Huang, K. Kong, and S. C. Park, J. High Energy Phys. 06 (2012) 099.

[75] T. G. Rizzo, Phys. Rev. D 86, 055024 (2012).

[76] T. Flacke, A. Menon, and D. J. Phalen, Phys. Rev. D 79, 056009 (2009).

[77] A. Datta, U. K. Dey, A. Shaw, and A. Raychaudhuri, Phys. Rev. D 87, 076002 (2013).

[78] A. Datta, K. Nishiwaki, and S. Niyogi, J. High Energy Phys. 11 (2012) 154.

[79] T. Flacke, A. Menon, and Z. Sullivan, Phys. Rev. D 86, 093006 (2012).

[80] S. K. Majee and S. C. Park, arXiv:1301.6421.

[81] T. Flacke, K. Kong, and S. C. Park, J. High Energy Phys. 05 (2013) 111.

[82] F. J. Petriello, J. High Energy Phys. 05 (2002) 003.

[83] N. Maru, T. Nomura, J. Sato, and M. Yamanaka, Eur. Phys. J. C 66, 283 (2010).

[84] K. Nishiwaki, J. High Energy Phys. 05 (2012) 111.

[85] S. K. Rai, Int. J. Mod. Phys. A 23, 823 (2008).

[86] G. Belanger, A. Belyaev, M. Brown, M. Kakizaki, and A. Pukhov, Phys. Rev. D 87, 016008 (2013).

[87] U. K. Dey and T. S. Ray, arXiv:1305.1016.

[88] T. Appelquist and H.-U. Yee, Phys. Rev. D 67, 055002 (2003).

[89] G. Bhattacharyya, S. Goswami, and A. Raychaudhuri, Phys. Rev. D 66, 033008 (2002).

[90] C. S. Lim, N. Maru, and K. Nishiwaki, Phys. Rev. D 81, 076006 (2010).

[91] A. Denner, Fortschr. Phys. 41, 307 (1993).

[92] K. R. Dienes, E. Dudas, and T. Gherghetta, Phys. Lett. B 436, 55 (1998).

[93] K. R. Dienes, E. Dudas, and T. Gherghetta, Nucl. Phys. B537, 47 (1999).

[94] G. Degrassi, S. Vita, J. Elias-Miró, J. R. Espinosa, G. F. Giudice, G. Isidori, and A. Strumia, J. High Energy Phys. 08 (2012) 098.
[95] S. Alekhin, A. Djouadi, and S. Moch, Phys. Lett. B 716, 214 (2012).

[96] J. Beringer et al. (Particle Data Group), Phys. Rev. D 86, 010001 (2012).

[97] G. Bhattacharyya, A. Datta, S.K. Majee, and A. Raychaudhuri, Nucl. Phys. B760, 117 (2007).

[98] A. Cornell and L.-X. Liu, Phys. Rev. D 84, 036002 (2011).

[99] M. Blennow, H. Melbeus, T. Ohlsson, and H. Zhang, Phys. Lett. B 712, 419 (2012).

[100] A. Datta and S. Raychaudhuri, Phys. Rev. D 87, 035018 (2013).

[101] T. Ohlsson and S. Riad, Phys. Lett. B 718, 1002 (2013).

[102] M. Blennow, H. Melbeus, T. Ohlsson, and H. Zhang, J. High Energy Phys. 04 (2011) 052.

[103] A. Cornell and L.-X. Liu, Phys. Rev. D 83, 033005 (2011).

[104] F. Jegerlehner, M. Y. Kalmykov, and B. A. Kniehl, Phys. Lett. B 722, 123 (2013).

[105] P. P. Giardino, K. Kannike, M. Raidal, and A. Strumia, Phys. Lett. B 718, 469 (2012).

[106] T. Abe, R. Kitano, Y. Konishi, K.-y. Oda, J. Sato, and S. Sugiyama, Phys. Rev. D 86, 115016 (2012).

[107] M.E. Peskin and T. Takeuchi, Phys. Rev. Lett. 65, 964 (1990).

[108] M. E. Peskin and T. Takeuchi, Phys. Rev. D 46, 381 (1992).

[109] M. Baak, M. Goebel, J. Haller, A. Hoecker, D. Kennedy, R. Kogler, K. Mönig, M. Schott, and J. Stelzer, Eur. Phys. J. C 72, 2205 (2012).

[110] H.-C. Cheng, K. T. Matchev, and M. Schmaltz, Phys. Rev. D 66, 036005 (2002).

[111] T. Flacke, D. Hooper, and J. March-Russell, Phys. Rev. D 73, 095002 (2006).

[112] I. Gogoladze and C. Macesanu, Phys. Rev. D 74, 093012 (2006).

[113] A. Arbey, G. Cacciapaglia, A. Deandrea, and B. Kubik, J. High Energy Phys. 01 (2013) 147.

[114] B. A. Dobrescu, D. Hooper, K. Kong, and R. Mahbubani, J. Cosmol. Astropart. Phys. 10 (2007) 012.

[115] T. Kakuda, K. Nishiwaki, K.-y. Oda, N. Okuda, and R. Watanabe, arXiv:1202.6231.

[116] K. Desch (Higgs Working Group of the Extended ECFA/ DESY Study), arXiv:hep-ph/0311092.

[117] S. Heinemeyer et al., Proceedings of Snowmass 05, econf C0508141, PLEN0044 (2005).

[118] T. L. Barklow, arXiv:hep-ph/0312268.

[119] T. Cheng, E. Eichten, and L.-F. Li, Phys. Rev. D 9, 2259 (1974).

[120] G. Passarino and M. Veltman, Nucl. Phys. B160, 151 (1979). 